\title{
The star cluster population of the spiral galaxy NGC $3370^{\star}$
}

\author{
M. Cantiello ${ }^{1}$, E. Brocato ${ }^{1}$, and J. P. Blakeslee ${ }^{2}$ \\ 1 INAF-Osservatorio Astronomico di Teramo, via M. Maggini snc, 64100 Teramo, Italy \\ e-mail: [cantiello; brocato]@oa-teramo.inaf.it \\ 2 Herzberg Institute of Astrophysics, National Research Council of Canada, 5071 W. Saanich Rd, Victoria, BC V9E 2E7, Canada \\ e-mail: john.blakeslee@nrc.ca
}

Received 12 March 2009 / Accepted 10 June 2009

\begin{abstract}
Aims. We study the photometric and structural properties of the star cluster system in the late type Sc spiral NGC 3370. Methods. BVI observations from the Advanced Camera for Surveys on board of HST are used to analyse in detail the colours, magnitudes and spatial properties of cluster candidates. The final catalogue of sources used for the study is composed by 277 objects. Results. The colour distributions of cluster candidates appear multi-modal. Although firm age constraints need the use of more agesensitive indicators, the comparison of cluster candidate colours with the colours of Galactic and Magellanic Clouds star clusters, suggests an age difference between the various sub-peaks, with a red old sub-system, a rich population of intermediate age ( $\sim 1 \mathrm{Gyr})$, and a blue tail of very young (below $\sim 100 \mathrm{Myr}$ ) clusters. The luminosity functions appear normal for this type of galaxy, as for the distribution of cluster effective radii $\left(R_{\mathrm{eff}}\right)$. Our analysis suggests the presence of a peak in the $R_{\mathrm{eff}}$ distribution at $\sim 3.0 \mathrm{pc}$, with blue (likely young) cluster candidates showing smaller radii respect to red (likely old) objects. Finally, inspecting the properties of candidate globular clusters, we find a colour distribution matching with Galactic Globulars, with a median $[\mathrm{Fe} / \mathrm{H}] \sim-1.5 \mathrm{dex}$, though a non negligible tail towards lower metallicities is also present.
\end{abstract}

Key words. galaxies: individual: NGC 3370 - galaxies: star clusters

\section{Introduction}

The study of star clusters in galaxies provides an accurate and relatively straightforward tool to unveil the mechanisms that produced the present organisation and evolutionary properties of stars in the galaxy. Star clusters allow to inspect the host galaxy both as a system and as single star clumps hosting - relatively simple stellar populations. Moreover, stellar clusters are the cradle of a large fraction of stars (e.g. Lada \& Lada 2003, and references therein). As a consequence, understanding the properties of the Star Cluster (SC hereafter) system in a galaxy helps understanding the properties of the host galaxy. Furthermore, SCs are relatively simple stellar systems - in first approximation single age and single metallicity stellar populations - much simpler than the stellar system of the host galaxies, providing the natural bridge from local single star analysis to distant unresolved stellar population studies.

Motivated by the chance to analyse the evolutionary properties of the host galaxy, in the last decade, especially after the HST refurbishment, a great deal of work has been dedicated to the study the properties (luminosity, radius, mass, etc.) of the SC system in spiral and irregular galaxies (e.g. Whitmore \& Schweizer 1995; Whitmore et al. 1999; Larsen \& Richtler 2000; Bik et al. 2003; de Grijs et al. 2003; Gieles et al. 2006a,b, just to quote few examples).

In this work we present a study of the SC system in the spiral galaxy NGC 3370, based on archival multi-band images taken with the HST Advanced Camera for Surveys.

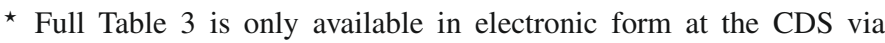
anonymous ftp to cdsarc.u-strasbg.fr $(130.79 .128 .5)$ or via http://cdsweb.u-strasbg.fr/cgi-bin/qcat?J/A+A/503/87
The galaxy is a nearly face on Sc spiral (Hubble type SA(s)c, de Vaucouleurs et al. 1991), showing an intricate tangle of arms, and mass similar to our own Milky Way. NGC 3370 was the host of the type Ia Supernova SN 1994ae (van Dyk et al. 1994). The light-curve of this SN Ia is one of the nine templates used by Riess et al. (1996) to introduce the SNe Ia multicolour lightcurve shapes method to estimate distances. With the advent of the high efficiency ACS camera on board of HST, and thanks to the specific properties of the galaxy (face on, relatively nearby spiral), NGC 3370 has been targeted by an HST program to study the light-curves of Cepheids, to provide a direct calibration of the SN Ia distance (Riess et al. 2005). The program, carried out on optical $V$ and $I$ images, has been integrated by complementary $B$ band exposures for the Hubble Heritage program.

The optical $B V I$ data available offer an excellent opportunity to investigate accurately not only the photometric, but also the structural properties of the SC systems in this spiral, thanks to the high resolution and small PSF FWHM of the ACS.

The paper is organised as follows. In Sect. 2 we present the observational dataset used, the data analysis carried out, and the detection and selection criteria chosen for SC candidates. The study of photometric and spatial properties and the comparison with models is discussed in Sect. 3. We present our conclusions in Sect. 4.

\section{Observations, data analysis and star cluster selections}

The ACS/WFC camera observations of NGC 3370 used in this work come from two different proposals. The proposal ID \#9351 (P.I. A. Riess) consists of very deep $F 555 W(\sim V)$ and $F 814 W$ 


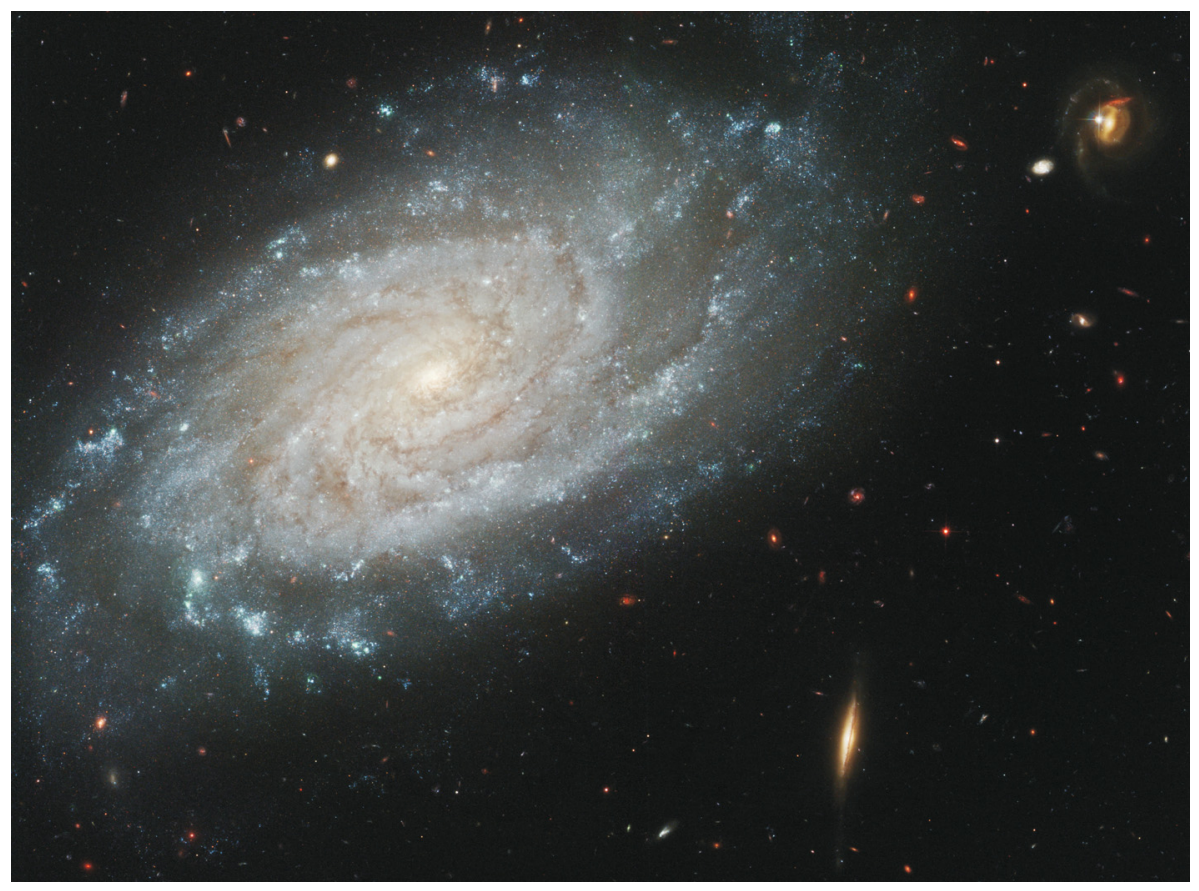

Fig. 1. ACS $B, V$ and $I$ composite image of NGC 3370, the image size is roughly $3.5 \times 2.5$ arcmin (Credits: NASA, The Hubble Heritage Team and A. Riess - STScI).

Table 1. NGC 3370 properties and observations.

\begin{tabular}{ll}
\hline \hline RA \& Dec (J2000) & $0^{\mathrm{h}} 47^{\mathrm{m}} 04.0^{\mathrm{s}}+17^{\mathrm{d}} 16^{\mathrm{m}} 25^{\mathrm{s}}$ \\
Morphological Classification & $\mathrm{Sc}^{a}$ \\
Morphological T-type & $5.0 \pm 1.1^{a}$ \\
Total corrected $B$ magnitude & $\sim 11.7^{a}$ \\
$E(B-V)$ & $0.031^{b}$ \\
Distance Modulus & $32.29 \pm 0.06^{c}$ \\
$F 435 W$ Exposure time (s) & 9600 \\
$F 555 W$ Exposure time (s) & 57600 \\
$F 814 W$ Exposure time (s) & 24000 \\
\hline
\end{tabular}

${ }^{a}$ Data from Hyperleda (http://leda. univ-lyon1. fr/).

${ }^{b}$ Extinction from Schlegel et al. (1998). ${ }^{c}$ Riess et al. (2005).

band $(\sim I)$ observations to study the Period-Luminosity relation of the Cepheids in the galaxy (Riess et al. 2005), with the purpose of deriving the distance of NGC 3370 and provide a precise luminosity calibration of the Type Ia supernova observed in the galaxy. The study realised by Riess et al. provided the farthest direct measurement of Cepheids. The other set of $F 435 \mathrm{~W}$ band $(\sim B)$ images was obtained for the Hubble Heritage (proposal ID \#9696, P.I. K. Noll). A colour image of the galaxy is shown in Fig. 1. The main properties of NGC 3370, and of the observational dataset used are reviewed in Table 1. For the distance modulus, we adopt Riess et al. value, $\mu_{0}=32.29 \pm 0.06$, derived from an empirical calibration of Cepheids' Period-Luminosity relation, and an LMC distance modulus of $18.50 \mathrm{mag}$.

Standard reduction was carried out on raw images using the APSIS image processing software (Blakeslee et al. 2003). Since ACS/WFC images are under-sampled for wavelengths at $\lambda \lesssim$ $11000 \AA$, and thanks to the fine dithering of the input images, a resolution better than the ACS detector pixels size, $0.05^{\prime \prime} / \mathrm{pixel}$, can be achieved with these images. We adopted a resampling of $0.035^{\prime \prime} /$ pixel as input parameter for APSIS, which corresponds to $\sim 5 \mathrm{pc} / \mathrm{pixel}$ at the galaxy distance.

After cosmic-ray rejection, alignment, drizzling, and final image combination, we derived the sky background from the median counts in the image corner with the lowest number of

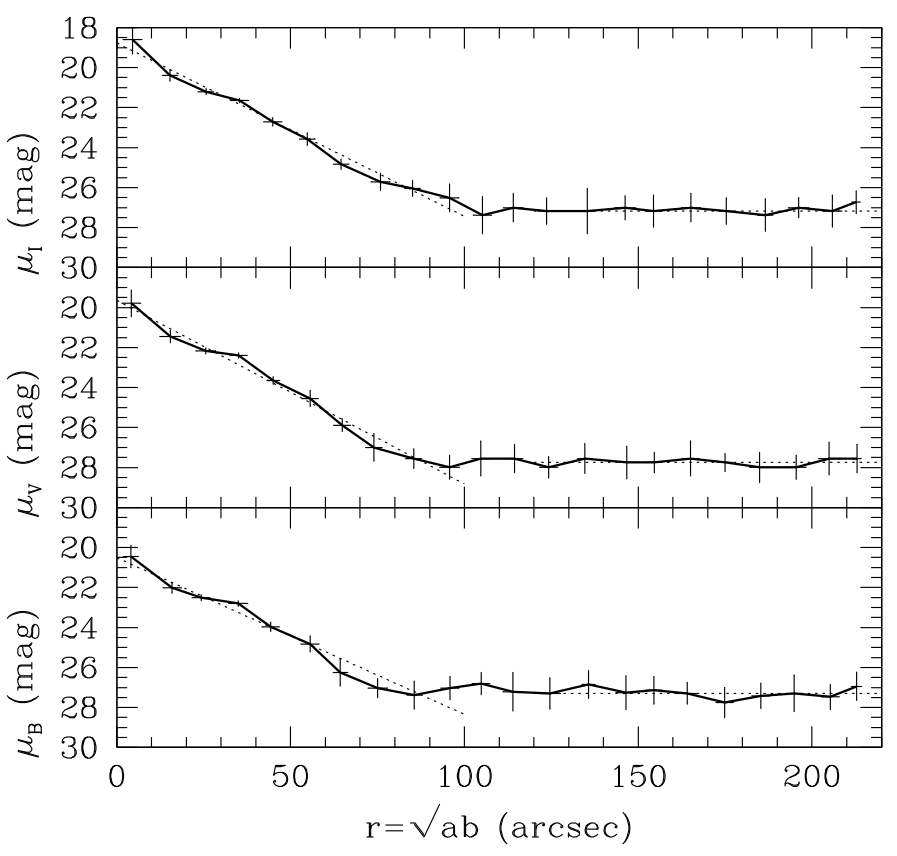

Fig. 2. Upper to lower panel: radial $I, V$ and $B$ band surface brightness profiles of NGC 3370. The linear fit to the data at $r \lesssim 100^{\prime \prime}$ is shown with a dotted line. The horizontal dotted line at $r \geq 120^{\prime \prime}$ shows the background level in the three observed bands.

counts. This assumption is supported by the fact that the surface brightness profile of the galaxy is practically constant for $r \gtrsim 120^{\prime \prime}$ (Fig. 2).

The source detection was obtained using SExtractor (Bertin \& Arnouts 1996) independently on the $V$ and $I$ frames. Due to the low $S / N$ of $B$-band images, the source detection in this band was done using SExtractor in the pixel association mode (ASSOC mode), adopting as reference the coordinates of sources detected in the $I$-band frame. We used a minimum of 5 connected pixels and $2 \sigma$ threshold for sources detection. This resulted in a list of $\sim 20000$ objects. The coordinates of sources 
detected from $V$ and $I$ frames were cross-checked, allowing a maximum wandering of 4 pixels $^{1}$, then we performed a first very bland selection of SC candidates based on the SExtractor parameters star/galaxy classification, object major-to-minor axis ratio, and $F W H M$ (object with CLASS_STAR $=0.00$, elongation A_IMAGE/B_IMAGE $\geq 2.5$ and $F W H M>12$ were rejected). Moreover, we rejected the sources fainter than $m_{I}=27.5$, and $m_{V}=28.5 \mathrm{mag}^{2}$. Using such limits we ended up with a list of $\sim 8000 \mathrm{SC}$ candidates whose structural properties were then analysed.

Given the linear pixel scale of these NGC 3370 images, and the ACS Point Spread Function FWHM of $\sim 2.5$ pixels, we are in the condition of using the ishape software (Larsen 1999) to estimate the spatial extension of SC candidates. This software package allows to determine the physical characteristics of SC candidates ( $R_{\text {eff }}$, elongation, etc.) by convolving analytic profiles with the PSF, then best-fit parameters are obtained by the $\chi^{2}$ minimisation. Ishape provides accurate intrinsic $F W H M$ values of slightly resolved objects down to $1 / 10$ the $F W H M$ of the PSF, corresponding to $\sim 1.2 \mathrm{pc}$ in our case. In other words, provided that a good PSF sampling can be obtained, effective radii of star clusters $\gtrsim 1.2 \mathrm{pc}$ can be retrieved. The input PSF to be used for ishape needs to be subsampled by a factor 10 with respect to the input science images. As suggested by the i shape handbook, we generated our PSF using the SEEPSF task of DAOPHOT within IRAF ${ }^{3}$. To avoid contamination from clusters, the list of stars to determine the PSF was selected on the basis of the FWHM, colours and magnitudes from DAOPHOT. We finally used 10 bright stars (all common to the catalogue of comparison stars in Table 3 of Riess et al.), evenly distributed on NGC 3370, and all at distances $>50^{\prime \prime}$ from the centre of the galaxy. It is useful to anticipate here that a posteriori all the objects used as template for the PSF, and all the Cepheids detected by Riess et al. were catalogued as stars by ishape.

Among the various profiles available with ishape, we choose a Moffat profile with a power law index -2.5 , since it gave generally better residuals compared to other choices. A Moffat profile has already been shown to be effective in fitting the profiles of both young and old star clusters, though with slightly smaller exponents with respect to our choice (see, e.g., Elson et al. 1987; Larsen 1999; Mackey \& Gilmore 2003). As discussed in Larsen (1999), the details of the model profile chosen are not an issue as long as the the $R_{\text {eff }}$ values are derived

\footnotetext{
1 The alignment between single HST frames is generally better than the maximum wandering adopted here. Our choice has been motivated by the large spatial variability of NGC 3370 surface brightness profile, and its wavelength dependence, which may lead to different estimates of the source centroid in different bands. A posteriori, we verified that for none of the SC candidates in the final sample the coordinate wandering between $V$ and $I$ frames exceeds $\sim 2$ pixels.

2 The aim of the selection based on the SExtractor morphological parameters CLASS_STAR, and A_IMAGE/B_IMAGE is to remove from the candidate list all obvious non SCs. Visual inspection of objects rejected in this way revealed that they are mostly extended, irregular, diffuse or elongated objects. Moreover, in a further selection based on ishape output parameters (see end of this section), we rejected candidates with elongation estimated by ishape $\geq 2$. The limiting magnitudes adopted here are, instead, related to the $S / N$ ratio of ishape. Tests made on sources fainter than the limiting magnitudes adopted showed that the $S / N$ is in all cases much lower than the minimum acceptable value $\sim 30$ suggested by Larsen (1999).

${ }^{3}$ IRAF is distributed by the National Optical Astronomy Observatories, which are operated by the Association of Universities for Research in Astronomy, Inc., under cooperative agreement with the National Science Foundation.
}

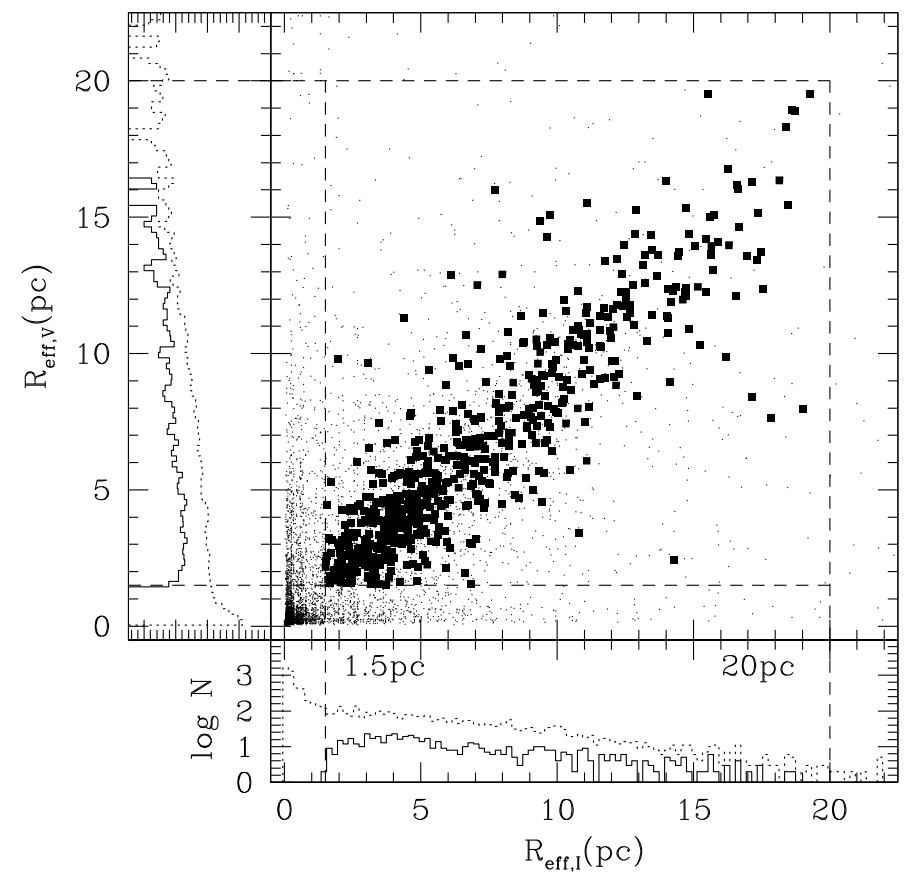

Fig. 3. Central panel: effective radii from the $I$-band frame versus the $V$-band $R_{\text {eff }}$ (dots). The full squares show cluster candidates whose $R_{\text {eff }}$ agree within $2 \sigma$ in both bands (see text for details). The lower and left panels show the histograms of radii for the whole sample of sources (dotted lines), and for the SC candidates with matching radii (solid line). The dashed lines show the $1.5 \mathrm{pc}$ and $20 \mathrm{pc}$ cut radii.

for sources with intrinsic sizes smaller or similar to that of the PSF, as in the present case. The analysis was carried out independently on the I, V, and B frames, although only $I$ and $V$ band data were finally used to estimate the average effective radii of SC candidates.

The ishape output parameters were used to further constrain the list of SC candidates. In particular, we reject the objects:

- whose effective radii $R_{\text {eff }}$ differ more than $2 \sigma$ in the two bands (Fig. 3). The $\sigma$ are derived using standard error propagation on ishape fitted parameters and uncertainties. In few cases the output of the fit is very good in both bands, so that the rms is small for $V$ and $I R_{\text {eff }}$, and the object will end up rejected by a simple $2 \sigma$ rejection criterion. However, if the systematic uncertainties arising, for example, from the PSF, the analytic profile chosen, etc., are taken into account, then the two sources should be included in the catalogue. After several tests, we decided to use a default systematic uncertainty of $\sim 15 \% R_{\mathrm{eff}}$. Then, for the $2 \sigma$ selection we used the maximum $\sigma$ between the $r m s$ from ishape, and $\sim 15 \% R_{\text {eff }}$; - with $S / N_{\text {ishape }} \lesssim 30$ (Larsen 1999);

- with too small/large $R_{\text {eff }}$. The specific properties of Moffat profile chosen allow to estimate effective SC radii below the $\sim 1.2$ pc limit aforementioned (see Larsen 1999, and ishape user's guide). However, we choose to adopt as lower and upper rejection limits $R_{\mathrm{eff}}<1.5 \mathrm{pc}$, and $R_{\mathrm{eff}}>20 \mathrm{pc}$, respectively. As a reference, using the data from McLaughlin \& van der Marel (2005), 1\% of massive star clusters in the Milky Way and its satellites have $R_{\mathrm{eff}}<1.5 \mathrm{pc}$, while $\sim 3 \%$ have $R_{\text {eff }}>20$ pc;

- elongation (major to minor axis ratio) $>2.0$, comparable to the maximum observed in Magellanic Clouds star clusters (van den Bergh \& Morbey 1984). 

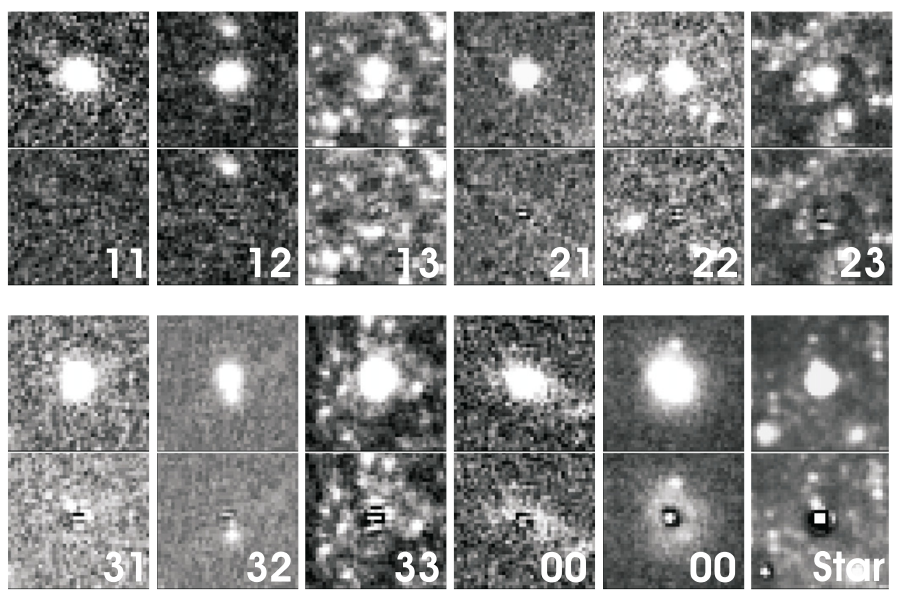

Fig. 4. Some examples of visual classifications codes. For each source we show the original $I$-band image (upper sub-panels) and the ishape residuals (lower sub-panels). Objects associated to the classes 33 (large residuals, crowded area), 100 (possible background galaxies), and stars are rejected in the final catalogue of SC candidates.

With these selection criteria we obtained a list of $\sim 630$ SC candidates. It is worth to emphasise the strongest constraints come from the $S / N$ limit, and the $R_{\text {eff }}$ (most of the $\sim 8000$ sources are actually classified as stars, see Fig. 3 ).

To verify the goodness of the ishape best fit parameters we have visually inspected all sources, introducing a subclassification of sources depending on the residuals of SC candidates subtraction, and on the crowding. The visual classification code was used for further selections. Figure 4 shows some examples of sources with different visual classification. The visual class code that we used is a number composed by two digits, the first associated to the goodness of i shape residuals, the second to the level of crowding. For both digits the range adopted is $1-3$. For the first digit $1 / 2 / 3$ means good/satisfactory/large residuals, respectively. The second is related to crowding with $1 / 2 / 3$ meaning no crowding/less/more than three sources within 20 pixels from the SC candidate. As an example visual class $=$ 12(31) means good residuals and 1 or 2 sources within 20 pixels (large residuals and no other source within 20 pixels). A default visual class $=100$ is adopted for diffuse objects (likely background galaxies) showing very large residuals in the ishape subtracted frame (see Fig. 4).

The aperture photometry of all the sources selected has been obtained using the Phot/Apphot task under IRAF. Due to the high, strongly variable background in some regions of the galaxy, we made different tests using 3,5 and 7 pixels aperture radius, and 10, 16 pixel annulus for background determination. As a final choice, we adopted 3 pixels aperture for the photometry, while the background was estimated in an annulus 16 pixels inner radius and 3 pixel width.

Dealing with slightly extended objects having different radii, the aperture correction depends on the radius of the cluster. As a consequence, we fitted the aperture correction versus $R_{\text {eff }}$. To avoid contamination from galaxy background, uncertain $R_{\text {eff }}$ estimates, and crowding, we decided to use only sources brighter than $m_{I, 3 \mathrm{pix}}=26.5 \mathrm{mag}$, ishape $S / N>50$, visual class $\leq 23$, and local surface brightness background $\mu_{V} \geq 22.5$ mag (galactocentric radius $\left.R_{\mathrm{gc}} \gtrsim 35^{\prime \prime}\right)$. Aperture magnitude was derived in 3 and 6 pixels $(\sim 15$ and $30 \mathrm{pc})$ apertures over the selected sources. Figure 5 shows the aperture correction derived as the difference between 3 and 6 pixels aperture magnitudes, versus the effective radii. The best fit linear correlations shown in the

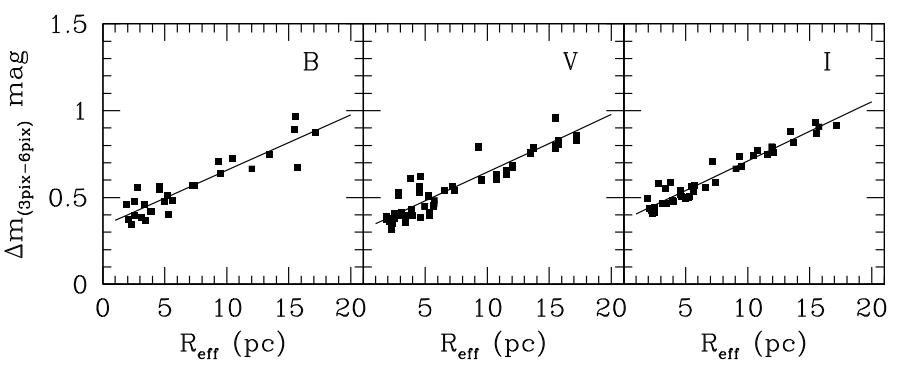

Fig. 5. Aperture corrections in $B, V$, and $I$-band versus the $\mathrm{SC}$ candidate radius. The solid lines show the best fit linear equation.

figure for each passband was then used to correct the magnitudes of all SC candidates in $B$-, $V$-, and $I$-band.

The correction from 6 pixels to "infinite" radius, as well as the transformations from the $F 435 W / F 555 W / F 814 W$ ACS filter system to standard $B / V / I$ filters, and foreground extinction, were applied following the prescriptions by Sirianni et al. (2005).

Once all corrections were made, we added new constraints to further clean the final list of SC candidates. In particular, to eliminate objects with very high internal extinction and possible distant background galaxies, we considered only those sources with $V-I \leq 1.5 \mathrm{mag}, B-V \leq 1.2 \mathrm{mag}$ (typical maximum colours for globular clusters), and a maximum photometric uncertainty $\Delta(V-I) \leq 0.15 \mathrm{mag}$. SC candidates showing large residuals and in crowded regions, candidate background galaxies (visual class $=33$ and 100 , respectively) as well as sources located in regions with strongly variable background (to avoid objects with photometry significantly contaminated by internal extinction) have been rejected, too. The final list of SC candidates matching with the required constraints is composed by 277 objects $^{4}$. A schematic view on the effects of the various selection criteria applied, with more details on the numbers, is reported in Table 2. The table gives the number of objects before $\left(N_{\text {in }}\right)$ and after $\left(N_{\text {out }}\right)$ each selection criteria is applied, together with the list of criteria adopted and the number of candidates rejected with the chosen parameter $\left(N_{\text {rej }}\right)$. It is worth noting that the same object might satisfy two or more rejections, thus the total number of objects rejected, $N_{\text {rej, tot }}$, is not simply obtained by summing up the numbers in the $N_{\text {rej }}$ lines. The positional, photometric and structural properties of the final catalogue of sources are reported in Table 3.

\section{Discussion}

\subsection{Analysis of colours}

Figure 6 shows the colour-colour diagram of the final catalogue of SC candidates. In the figure it is shown also the effect of the various steps of the selection. Dark-grey dots, empty and filled circles mark the position of the sample after the selections based on SExtractor, ishape, and photometric parameters, respectively. The full circles in the figure refer to the final selected sample of SC candidates. Also shown in the figure, with a grey area, is the region of the SPoT Simple Stellar Populations models for metallicity between $[\mathrm{Fe} / \mathrm{H}] \sim-2.5 \div 0.3 \mathrm{dex}$, and ages in the interval $25 \mathrm{Myr} \div 14 \mathrm{Gyr}$ (see Raimondo et al. 2005, for more details on SPoT SSP models). As expected, the data overlap to SSP models, since SC are, at least in first approximation, well represented by SSPs. It must be emphasised, however, that

\footnotetext{
4 The complete catalogues of sources can be retrieved upon request to the authors.
} 
Table 2. Number of objects rejected per selection criteria.

\begin{tabular}{|c|c|c|c|c|c|c|}
\hline $\begin{array}{l}\text { First selectio } \\
N_{\text {in }}=17767, \\
\text { Sel. Criteria } \\
N_{\text {rej }}\end{array}$ & $\begin{array}{c}\text { SExtractor selection cr } \\
N_{\text {out }}=8081, N_{\text {rej, tot }}=96 \\
\text { CLASS_STAR }=0.00 \\
675\end{array}$ & $\begin{array}{l}\text { eria } \\
6 \text { Elong.SExt. }_{1652} \geq 2.5 \\
\quad\end{array}$ & $\begin{array}{c}m_{I} \geq 27.5 \\
3251\end{array}$ & $\begin{array}{c}m_{V} \geq 28.5 \\
3962\end{array}$ & $\begin{array}{c}F W H M_{I} \geq 12 \\
2452\end{array}$ & $\begin{array}{c}F W H M_{V} \\
3287\end{array}$ \\
\hline $\begin{array}{l}\text { Second selec } \\
N_{\text {in }}=8081, \\
\text { Sel. Criteria } \\
N_{\text {rej }}\end{array}$ & $\begin{array}{c}\text { on: Ishape selection crit } \\
\text { out }=635, N_{\text {rej, tot }}=7446 \\
2 \sigma R_{\text {eff }} \\
1414\end{array}$ & $\begin{array}{c}\text { Elong }_{\cdot \text { ish. }}>2.0 \\
1293\end{array}$ & $\begin{array}{c}S / N<30 \\
5051\end{array}$ & $R_{\mathrm{eff}} \geq 1.5 \mathrm{pc}$ & $R_{\mathrm{eff}} \leq 20 \mathrm{pc}$ & \\
\hline $\begin{array}{l}\text { Third selecti } \\
N_{\text {in }}=635, N \\
\text { Sel. Criteria } \\
N_{\text {rej }}\end{array}$ & $\begin{array}{c}\text { n: photometric selection } \\
=277, N_{\text {rej, tot }}=358 \\
\Delta(V-I)>0.15 \\
17\end{array}$ & $\begin{array}{l}\text { riteria } \\
\text { vis. class } \geq 33 \\
286\end{array}$ & $\begin{array}{c}V-I \geq 1.5 \\
66\end{array}$ & $\begin{array}{c}B-V \geq 1.2 \\
19\end{array}$ & & \\
\hline
\end{tabular}

Table 3. Structural and photometric properties of selected SC candidates. The full table is only available in electronic form at the CDS.

\begin{tabular}{rrrrrrrrrrrrc}
\hline \hline ID & RA $(\mathrm{deg})$ & Dec $(\mathrm{deg})$ & $R_{\mathrm{gc}}\left({ }^{\prime \prime}\right)$ & $R_{\text {eff }}(\mathrm{pc})$ & \pm & $m_{V}$ & \pm & $B-V$ & \pm & $V-I$ & \pm & v.c. \\
\hline 5 & 161.7465 & 17.2766 & 71.65 & 2.04 & 0.25 & 25.39 & 0.01 & 0.23 & 0.02 & 0.28 & 0.01 & 21 \\
10 & 161.7527 & 17.2907 & 79.03 & 5.50 & 0.45 & 26.15 & 0.02 & 0.67 & 0.06 & 0.94 & 0.03 & 12 \\
13 & 161.7513 & 17.2858 & 69.83 & 4.68 & 0.92 & 25.40 & 0.02 & -0.04 & 0.03 & -0.09 & 0.05 & 23 \\
14 & 161.7552 & 17.2964 & 91.56 & 5.58 & 0.41 & 25.98 & 0.01 & -0.03 & 0.03 & 0.00 & 0.03 & 22 \\
15 & 161.7556 & 17.2980 & 96.25 & 13.67 & 0.21 & 24.57 & 0.01 & 0.51 & 0.01 & 1.30 & 0.01 & 21 \\
17 & 161.7537 & 17.2915 & 79.24 & 6.86 & 0.45 & 25.77 & 0.01 & 0.58 & 0.04 & 0.82 & 0.02 & 23 \\
18 & 161.7527 & 17.2879 & 71.40 & 6.62 & 0.18 & 24.46 & 0.01 & 0.28 & 0.01 & 0.58 & 0.01 & 22 \\
21 & 161.7506 & 17.2792 & 60.20 & 6.35 & 0.44 & 26.26 & 0.02 & 0.22 & 0.04 & 0.69 & 0.03 & 12 \\
22 & 161.7523 & 17.2850 & 65.42 & 2.13 & 0.38 & 25.09 & 0.01 & -0.09 & 0.02 & 0.03 & 0.01 & 22 \\
23 & 161.7545 & 17.2898 & 72.80 & 4.50 & 0.34 & 25.69 & 0.02 & 0.70 & 0.06 & 0.86 & 0.03 & 23 \\
24 & 161.7519 & 17.2828 & 61.81 & 4.00 & 0.45 & 25.76 & 0.02 & 0.17 & 0.04 & 0.56 & 0.02 & 23 \\
25 & 161.7530 & 17.2856 & 64.93 & 3.84 & 0.40 & 25.58 & 0.01 & 0.38 & 0.03 & 0.56 & 0.02 & 12 \\
29 & 161.7495 & 17.2750 & 60.90 & 3.89 & 0.46 & 26.04 & 0.01 & 0.30 & 0.03 & 0.40 & 0.02 & 22 \\
30 & 161.7550 & 17.2895 & 70.74 & 13.23 & 0.46 & 26.17 & 0.02 & 0.34 & 0.06 & 1.38 & 0.03 & 12 \\
33 & 161.7529 & 17.2837 & 60.83 & 3.06 & 0.10 & 25.40 & 0.01 & 0.18 & 0.03 & 0.39 & 0.02 & 23 \\
34 & 161.7573 & 17.2952 & 84.98 & 2.54 & 0.08 & 24.64 & 0.01 & 0.70 & 0.02 & 1.05 & 0.01 & 22 \\
36 & 161.7603 & 17.3021 & 105.49 & 2.74 & 0.57 & 25.90 & 0.01 & 0.42 & 0.02 & 0.39 & 0.01 & 22 \\
37 & 161.7549 & 17.2870 & 64.02 & 5.79 & 0.24 & 26.37 & 0.02 & 0.62 & 0.07 & 1.16 & 0.03 & 13 \\
\hline
\end{tabular}

such overlap is not a trivial result. In fact, in the selections described in the previous section, the only constraint on colour was on the maximum $V-I$ and $B-V$ allowed.

One interesting feature appearing in Fig. 6 is the nonnegligible number of objects located at $B-V \sim 0.4$ and $B-I \sim$ 1.6 (five pointed stars in the figure). After different counterchecks, we concluded that there is no obvious reason to reject these clusters from the final catalogue. As shown by the arrow in the panels, even an internal extinction $E(B-V) \sim 0.5 \mathrm{mag}$ would not shift these SC candidates in the region of SSP models. Moreover, such high extinction is unlikely since such objects do not appear obviously associated to regions of high internal extinction, while they are rather evenly distributed over the area of the galaxy. One possible interpretation of these objects is that they are intermediate age SCs $(0.3 \leq t(\mathrm{Gyr}) \leq 2)$. Within this age interval, the emission of Thermally Pulsating AGB stars (TP-AGBs) dominates the light emitted by the SC, in particular in the long-wavelength regime $(\gtrsim 8000 \AA$; see, e.g. Maraston 2005 , and references therein). However, the presence of stars in such evolutionary stage is strongly influenced by stochastic effects. As a consequence, the stochasticity due to the number of cool TP-AGBs can significantly affect the integrated colours of the stellar system. To highlight such behaviour, the insert panel in Fig. 6 shows the sample of selected SC candidates, and the "outliers" at $B-V \sim 0.4$ and $B-I \sim 1.6$ mag (five-pointed stars), together with the region where the recent SSP models by Raimondo (2009) for intermediate ages and $[\mathrm{Fe} / \mathrm{H}] \sim-0.4$ dex are distributed (dark-grey area). The new set of models used has been computed with an updated version of the SPoT code, paying particular attention to the statistical effects generated by Thermally Pulsating AGBs. In the figure, the dark-grey area shows the distribution of models when the stochastic effects due to TP-AGB stars are properly taken into account. As shown in the insert, these models predict the existence of the outliers (optical models have been provided as a private communication by Raimondo, for more details on models and near-IR colours see Raimondo 2009). In a following section we will discuss more in details the data to models comparison for the whole sample of SC candidates.

Figure 7 shows the colour magnitude diagrams and colour histograms for the SC candidates. It is not surprising that the whole population of SC candidates in NGC 3370 does not have a unimodal colour distribution, showing the presence of sub-peaks possibly associated to different SC sub-populations.

As a matter of fact, since NGC 3370 is a late type Sc spiral, it is expected that this class of galaxies is actively forming stars, and star clusters. Thus, populations of young/intermediate age $\mathrm{SC}$ are expected to host in the galaxy, in addition to the old population of Globular Clusters (GCs). To highlight this point, in the colour histograms shown in Fig. 7, we have added an arrow showing the median colours of Galactic GCs (GGCs, $(B-V)_{0}=0.73 \pm 0.12,(B-I)_{0}=1.65 \pm 0.12 \mathrm{mag}$ for $\sim 90 \mathrm{GGCs}$ with $M_{V} \lesssim-5.0 \mathrm{mag}$, data from Harris 1996). It is rather 


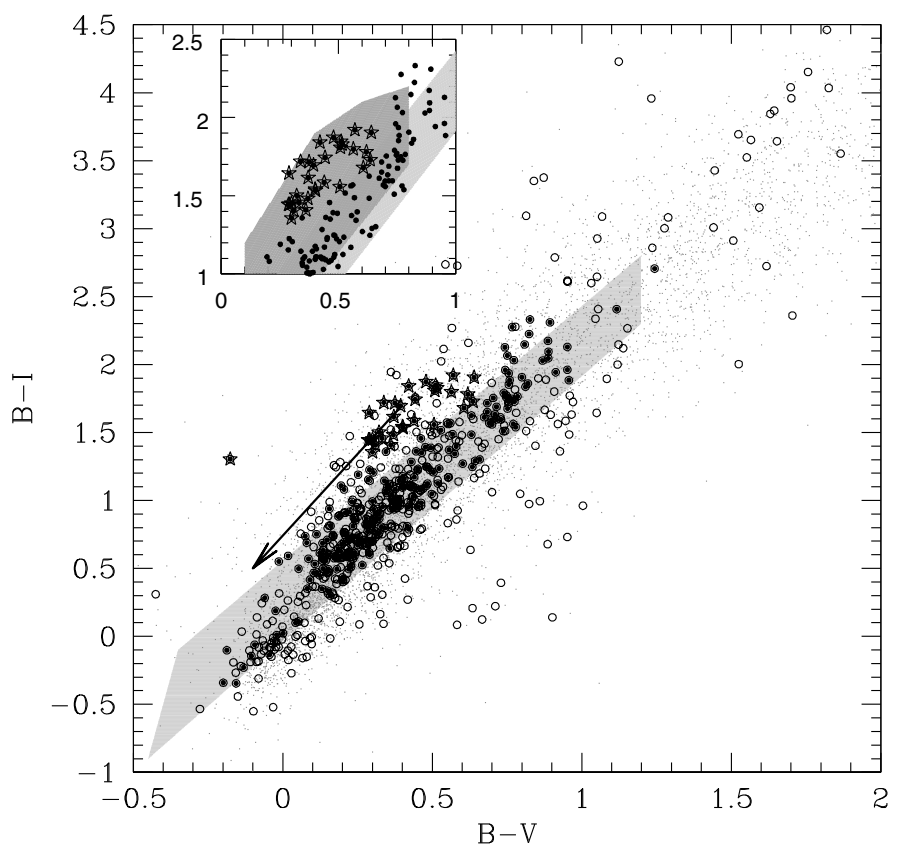

Fig. 6. Colour-colour diagram of the SC candidates. Dark-grey dots, empty and full circles mark the positions of the sample after the first, second and third (final catalogue) selections described in Table 2. The grey shaded area marks the region of SPoT-SSP models by Raimondo et al. (2005). The arrow shows the direction of the reddening vector. Five pointed stars are "outlier" SC candidates, possibly associated to intermediate age clusters. The insert panel shows the SC candidates (full dots), the SC outliers (five-pointed stars), and the standard SPoT SSP models (grey-shaded area). The dark-grey area shows the region occupied by SSP realisations of the recent SPoT models by Raimondo (2009), computed paying particular attention to the stochastic effects of TP-AGB stars. Only ages between $0.3-2 \mathrm{Gyr}$ and $[\mathrm{Fe} / \mathrm{H}] \sim-0.4$ dex are plotted (see text for details).

obvious, then, to conclude that the red peak observed in the lower panels is actually due to the GC population in the galaxy.

If the intermediate peak and the blue tail of SC candidates are taken into account, still using as reference the system of young open clusters (OCs) in the Galaxy, we find that the OCs at $B-V \sim 0.35$, and $B-I \sim 0.85 \mathrm{mag}$ have ages $\log t(\mathrm{yr}) \sim 8.4$ (based on only 3 Galactic OCs), while blue OCs at $B-V \sim$ -0.15 , and $B-I \sim-0.25 \mathrm{mag}$ have on average $\log t(\mathrm{yr}) \sim 7.0$ (based on $11 \mathrm{OCs})^{5}$. Given the results of such comparison with Galactic star clusters, it is reasonable to conclude that the colour peaks observed in Fig. 7 are mainly driven by age differences between the blue/intermediate/red SC candidates.

The situation, however, might be complicated by the possible presence of residual internal extinction. Since the galaxy is oriented nearly face on, the effect of internal extinction is minimised. Moreover, as discussed in previous section, to reduce possible extinction present in the dusty, star forming regions we rejected the sources located in areas of strongly varying background, or where dusty patches can be recognised. The presence of the colour peaks discussed above is an additional indirect probe against a possible strong contribute of internal reddening: a significant amount of dust extinction, in fact, should smear out such features. However, it is not possible to exclude at all the presence of some residual internal extinction. To have other indications about the possible nature of the colour range

\footnotetext{
5 Ages and colours for OCs are from the WEBDA database, available at http://www. univie.ac.at/webda
}

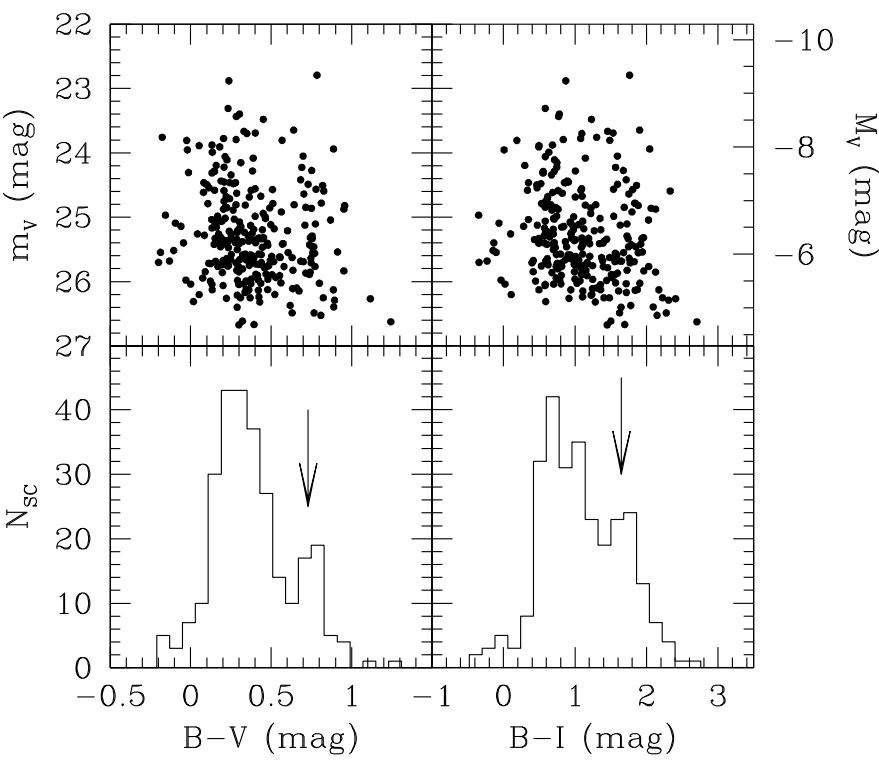

Fig. 7. Left panels: $B-V$ colour magnitude diagram (upper panel) and colour histograms (lower panel) of SC candidates. Right panels: as left, but for $B-I$. The arrows indicate the median colour of GGCs.

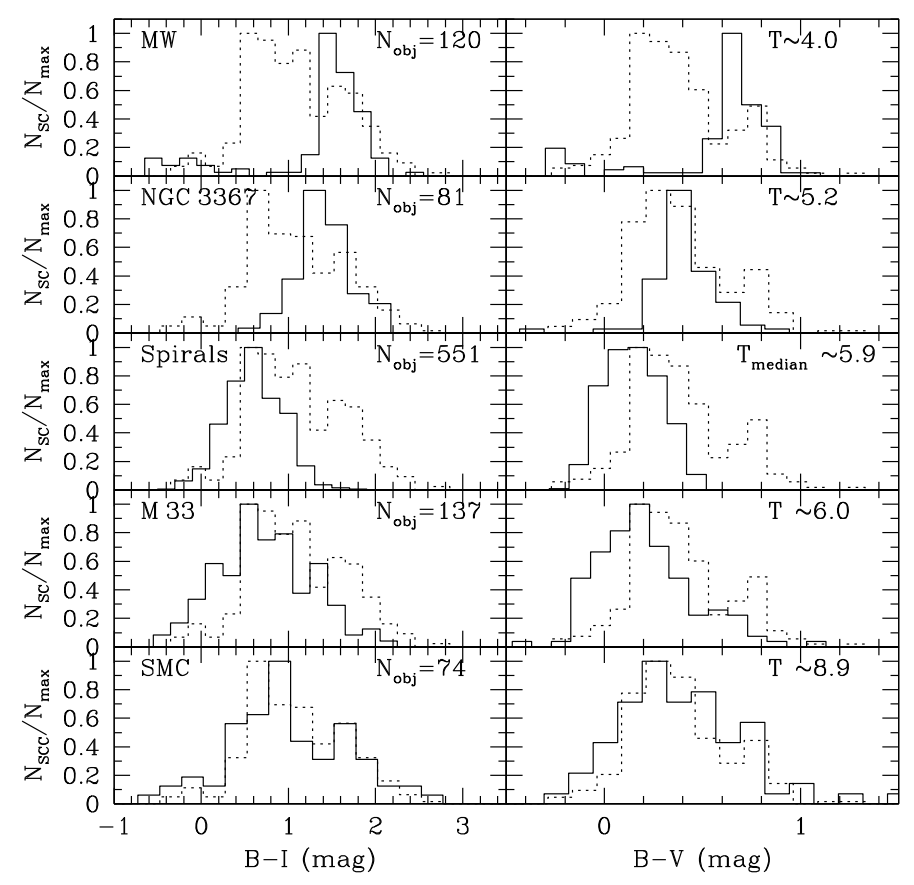

Fig. 8. Colour histograms of the comparison galaxies (as labelled in left panels). Dotted lines show NGC 3370 SC candidates. For NGC 3367 and SMC a different binwidth is used, because of the smaller number of SCs (see $N_{\text {obj }}$ labels in each panel).

and sub-peaks in NGC 3370, we compare the $B-I$ and $B-V$ colour histograms of SC candidates with the same data for other galaxies, some of which are corrected for internal reddening. In particular, we use the data from 1) Milky Way's GCs (Harris 1996), and Open Clusters (Lata et al. 2002); 2) NGC 3367 from García-Barreto et al. (2007); 3) the data of 20 Spirals from Larsen (1999); 4) M 33 data corrected for reddening (Park \& Lee 2007); 5) SMC Star Clusters from Rafelski \& Zaritsky (2005); 6) LMC data from Bica et al. (1996). All the data used are in (or are transformed to) the standard photometric BVI bands. The comparisons between different galaxies are shown in Figs. 8 


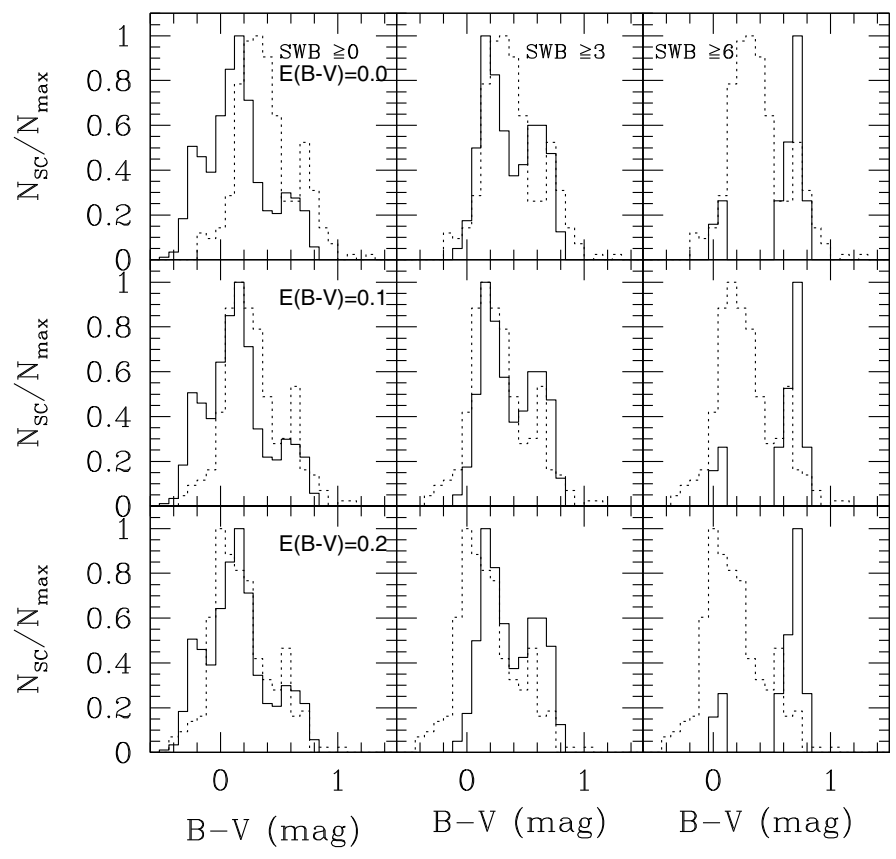

Fig. 9. $B-V$ colour histograms of LMC Star Clusters (solid lines) and NGC 3370 (dotted lines). The vertical blocks refer to LMC clusters selected according to the SWB class: the first, second and third row refer to LMC clusters with $S W B \geq 0,3,6$, respectively (see labels in upper panels). Horizontal blocks assume different average internal extinctions for NGC 3370 SC candidates: first, second and third row have $\left\langle E(B-V)_{\text {internal }}\right\rangle=0.0,0.1$, and $0.2 \mathrm{mag}$, respectively (see label in leftmost panels).

and 9. In order to compare only SCs in the luminosity range of the SC candidates considered here, we have excluded all SCs having total absolute magnitude $M_{V} \gtrsim-5 \mathrm{mag}$.

Figure 8 shows $B-I$ and $B-V$ colour histograms of the $S C$ in the MW, NGC 3367, the spirals from Larsen (1999), M 33 and the SMC. The galaxies are ordered according to their morphological T-Type, all histograms are normalised to have 1 in the most populated bin. In each panel of the figure the colour histograms of SC candidates in NGC 3370 are shown with dotted lines.

By inspecting the colour histograms it is easy to reveal similarities and differences between the system of SC candidates in NGC 3370 and in other galaxies. One example is represented by the red sub-peak, which appears quite similar to the red sub-peak in the colour distribution of SMC clusters. Using the age estimates by Rafelski \& Zaritsky (2005) we find that the 20 SMC clusters with $B-I \gtrsim 1.6$ mag have a median age $t \sim 5$ Gyr. The range of colours generally shown by other systems is quite similar to that of NGC 3370. The only exception is represented by the colour histograms of the SCs from Larsen (1999). However, the selection criteria used by Larsen exclude SCs with $B-V \geq 0.45$, which represents $\sim 45 \%$ of our sample of objects. In addition, the very blue/young stellar clusters are rejected by Larsen, adopting a lower limit to the $\mathrm{H}_{\alpha}-R$ colour. Thus, we are lead to conclude that, rather than a systematic difference between our data and Larsen's, the observed differences are due to the intrinsically different SCs selection. In any case, the peak of the distributions of Larsen's data appears similar to the main peak of NGC 3370 SCs, though slightly shifted towards bluer colours.

The SCs data of NGC 3367 - the galaxy of the sample morphologically more similar to NGC 3370 - do not show obvious multi-modal colour distributions. The range of colours, and the position of the main peak are roughly similar to NGC 3370. As for what concerns SMC clusters, the colour distributions span over the same range, and the peak of SC sub-systems seems to be recognised here as in NGC 3370.

We provide in Fig. 9 a separate comparison to LMC data. The reason for keeping LMC data aside is that, in this case, we can play with the age of the selected clusters by using the SWB class (Searle et al. 1980) of LMC clusters, and also make a test on the internal extinction in NGC 3370. The figure shows the $B-V$ colour histograms of LMC clusters, corrected for internal extinction, and NGC 3370 SC candidates. Each row of panels in the figure shows NGC 3370 cluster candidates dereddened adopting a different average internal extinction, $\left\langle E(B-V)_{\text {internal }}\right\rangle=0.0,0.1$ and $0.2 \mathrm{mag}$ from upper to lower panel, respectively. In addition, assuming the SWB classes from Bica et al. (1996), each column of panels is obtained assuming a lower limit to the SWB. Specifically, the first column includes all SWB classes, while the other two include only LMC clusters with $\mathrm{SWB} \geq 3$ and 6, respectively (labels in the uppermost panels). All panels show NGC 3370 colour histograms (dotted lines) shifted according to the internal reddening assumed, and the LMC clusters colour histograms using the SWB selection criteria (solid lines). Some hints on age distributions and, possibly, internal extinction of the SC candidates can be derived by a careful inspection of this figure. The upper panels show that if old LMC clusters are taken into account (SWB $\geq 6, t \gtrsim 10^{9} \mathrm{yr}$ ), there is a good match between NGC 3370 red peak and the old clusters in LMC. Thus, we have an additional indication that this population is, in fact, composed by old GC candidates. It is worth to notice, in the case of $\left\langle E(B-V)_{\text {internal }}\right\rangle \sim 0.1$ and $\mathrm{SWB} \geq 3$ (central panel in the figure), the similarity between the main colour peaks of the two galaxies. Clearly, because of the differences between the two galaxies, it is not possible to conclude whether or not such good matching is a consequence of an average internal extinction of $\sim 0.1 \mathrm{mag}$ on a SC system in NGC 3370 with an age distribution similar to LMC. It is, however, reasonable to take this value as an upper limit for $\langle E(B-$ $\left.V)_{\text {internal }}\right\rangle$. Furthermore, the lower panels, which assume larger internal extinction, show that such average reddening would move the main peak to a colour corresponding to ages of the order of $\log t(\mathrm{yr}) \sim 7.5$ - obtained taking MW OCs and LMC clusters ages as reference. Although young SCs are expected to be present in late type, actively star forming galaxies, it is not reasonable to conclude such predominance of very young objects. The position of the red peak is not an issue in the latter case, since it is expected that old clusters are, on average, less affected by dust extinction being far from actively star-forming regions. Thus, the shift of the red peak for $\left\langle E(B-V)_{\text {internal }}\right\rangle=$ $0.2 \mathrm{mag}$ is not probable. However, since this test is carried out using a common average value for internal extinction, we cannot rule out high extinction values on single bluer/younger cluster candidates.

In conclusion, the comparison of the colour histograms for NGC 3370 SC candidates with other SCs from literature shows that the range of colours appear normal with respect to similar class of galaxies. Clearly, the comparisons shown cannot be used to constrain ages, or any residual internal reddening, because of the intrinsic differences existing between the galaxies. However, we can give some limits, in particular, it is reasonable that NGC 3370 hosts three sub-populations of SC candidates: one with colours typical of old GCs, one with very blue colours, characterising a population of very young SCs, and a rich population of intermediate age SC candidates. This expectation is partly supported by the spatial distribution of the SC candidates 

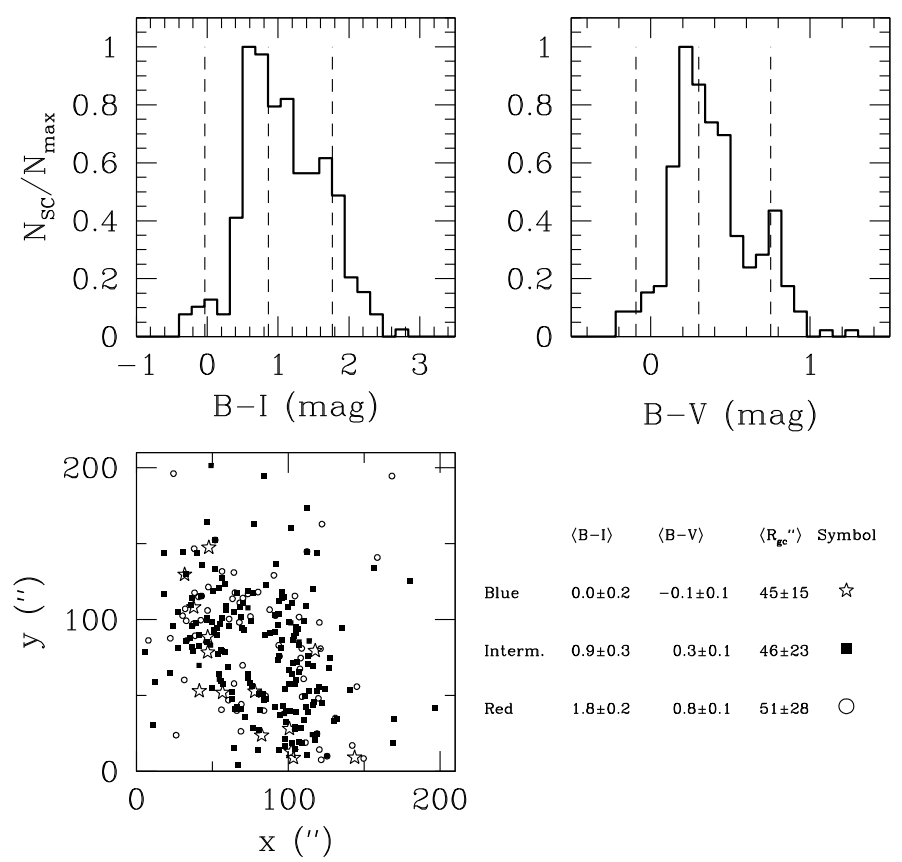

Fig. 10. Upper panels: colour histograms of SC candidates, the position of the three relative peaks is emphasised in each panel with dashed lines. Lower-left panel: spatial distribution SC candidates selected according to their membership to the colour sub-peaks (see text). Five pointed stars/full squares/circles mark candidates in the blue/intermediate/red tail respectively.

in the three colour peaks, shown in Fig. 10. In the figure we used the position of the three relative maxims to define a preliminary centre of each sub-peak in $B-I$ and $B-V$. Then, the median and standard deviation of the sole SCs within half-distance from the sub-peaks is obtained (dashed-lines in the upper panels). All SC candidates within $2 \sigma$ from the median value of the peak in both colours are then selected. The SCs selected in this way are shown in the lower-left panel of the figure: five pointed stars/full squares/circles mark candidates in the blue/intermediate/red tail respectively. By inspecting the spatial distribution of clusters, we recognise that the SC candidates in the blue tail are mostly located near the galactic centre, while the star clusters in the other colour bins are more spread over the galaxy, with the ones in the red peak being on average more distant from the centre of the galaxy, as expected for GC candidates. The median effective galactocentric distance of each SC class is estimated and reported in the figure.

\subsection{Luminosity functions}

Starting from the work by Elson \& Fall (1985) on LMC SCs, empirical data from a large sample of spiral and irregular galaxies have shown that the Star Cluster Luminosity Function (SCLF) usually follows a power law, with a sharp turnover at faint magnitudes. In this section we analyse the LF profile of the SC candidate system in NGC 3370.

To properly study the SCLF, we need to take into account the completeness corrections in the different bands. However, because of the selection criteria adopted, this is a complex task. In our case, in fact, the completeness functions should take into account $i$ ) the photometric completeness; ii) the selection on sizes, and iii) the visual identification. While the first two contributions to the completeness can be somehow obtained from numerical experiments, the third one is less straightforward to derive, if

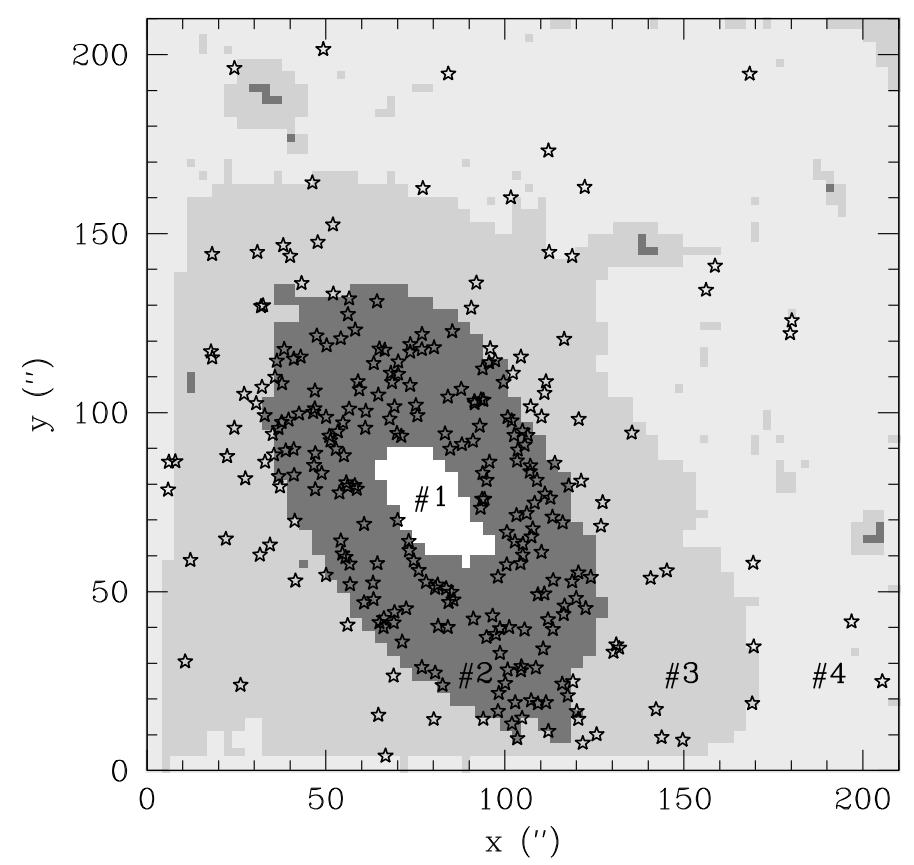

Fig. 11. Annuli division used to compute completeness functions. The regions are characterised by their median surface brightness: $\mu_{I}^{\# 1}<20$, $\mu_{I}^{\# 2}$ between $20 \div 23, \mu_{I}^{\# 3}$ between $23 \div 26.5$ and $\mu_{I}^{\# 4}>26.5 \mathrm{mag} / \operatorname{arcsec}^{2}$. Each annulus is labelled and identified with a different shade of grey. Also the loci of SC candidates are shown with five pointed stars. No SC candidate lies in the innermost annulus (\#1, white area).

feasible at all. Moreover, the completeness fraction is different for objects of different sizes. For such reasons we choose to adopt the following approach: the galaxy is divided in four concentric regions, selected on the basis of the median surface brightness of the galaxy. In particular, we choose $\mu_{I}^{\# 1}<20$, $20<\mu_{I}^{\# 2} \leq 23,23<\mu_{I}^{\# 3} \leq 26.5$, and $\mu_{I}^{\# 4}>26.5 \mathrm{mag} / \mathrm{arcsec}^{2}$ as separation between the regions \#1, \#2, \#3, and \#4. Figure 11 shows the four regions and the position of SC candidates. In each of the four annuli, then, we run completeness tests separately. To add artificial SCs we used DAOPHOT/ADDSTAR, adopting a template SC candidate derived from the best candidates in our list. Accurate completeness functions would need different numerical experiments obtained with SC templates of various $R_{\text {eff }}$. We have not carried out such detailed analysis. It is worth to remark, though, that the median size of template $\mathrm{SC},\left\langle R_{\mathrm{eff}}\right\rangle \sim 3 \mathrm{pc}$, is representative of the vast majority of the SC candidate population (see next section).

To derive completeness functions, SC candidates at fixed magnitudes are artificially added in the frame on a grid of 100 pixel separation both in $x$ and $y$ coordinates, the photometry of artificial sources is then obtained in the same way as for the original data. The comparison of the input and output photometric catalogues is used to evaluate the completeness fraction $f \equiv N_{\mathrm{SC} \text { detected }} / N_{\mathrm{SC} \text { added }}$. The experiments are repeated for i) different magnitudes (between 15 and $30 \mathrm{mag}$, with steps of $0.1 \mathrm{mag}$ ), and $\mathrm{ii}$ ) for different positions of the grid of stars. The results of the completeness tests are shown in Fig. 12. The completeness fraction is quite low in the innermost annulus (\#1), though we recall that no source in the final catalogue lies in such area of high, and highly variable galaxy background (Fig. 11).

Using the completeness functions so derived, we estimated the corrected LF of SC candidates, shown in Fig. 13. The observed sharp falloff is an artifact due to the sample incompleteness at faint magnitudes. In the three panels of the figure we 

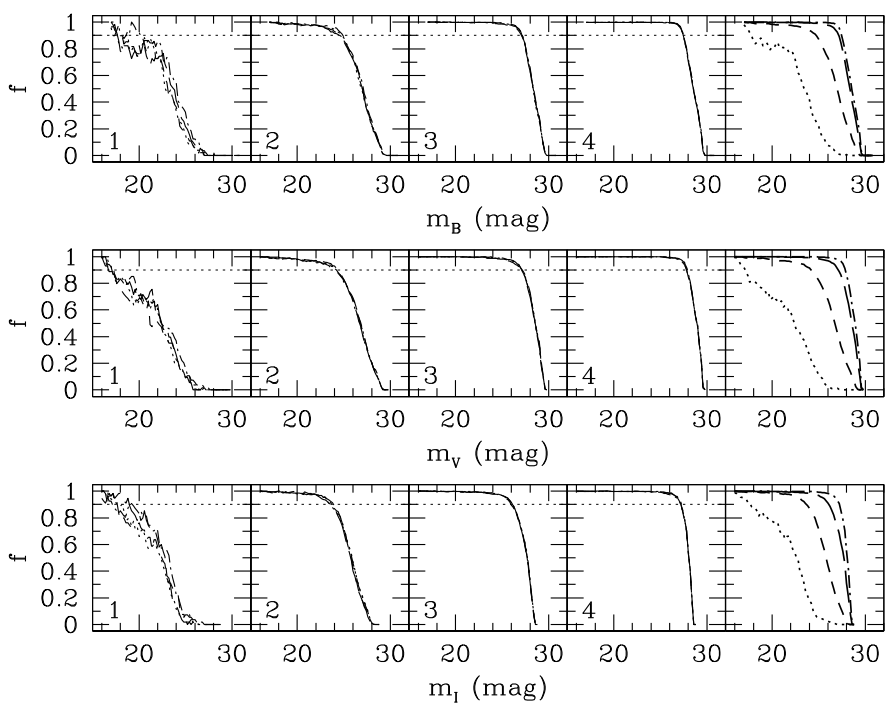

Fig. 12. $B, V$ and $I$-band completeness functions for the each of the four annuli considered (labelled in the panels). For each one of the 4 annuli, we have carried out several numerical experiments shifting the grid of artificial stars. Different experiments are shown with different line types in each panel. The horizontal dotted line marks the $90 \%$ completeness limit in the annulus. The rightmost panels show the median completeness function for each annulus and filter.

plot a fit to the data, the slope $\alpha$ obtained from a power law fit $\mathrm{d} N / \mathrm{d} L \propto L^{\alpha}$ is also reported. To minimise the effect of incompleteness, the upper limit of the fit is chosen so that the completeness fraction in the innermost useful annulus, \#2, is $f \gtrsim 0.5^{6}$. Notwithstanding the complex issue of completeness, the power law fit to the data provides exponents $\alpha \sim-2.2$ similar to what typically found in spirals and starburst galaxies (e.g. Miller et al. 1997; Whitmore et al. 1999; Larsen 2002; de Grijs et al. 2003). We also find, for all three passbands considered, steeper slopes on the bright sides of the LFs, with power law exponents as large as $\alpha \sim 3.0$. In all cases, however, the new exponent agrees within $1 \sigma$ uncertainty with the single power-law slopes reported in the figure. The presence of a bend in the SCLF has already been found in various galaxies. As discussed in details by Gieles et al. (2006a,b), a SC system originating from a truncated mass function $(\mathrm{MF})$, possibly caused by a truncated MF in the giant molecular clouds, is expected to show such a bend, with steeper slopes on bright side of the LF. One other prediction of a truncated MF is the steepening of the LF at longer wavelengths. In spite of the uncertainties on the fitted slopes, this behaviour seems to be found in our data. Nevertheless, comparing maximum cluster luminosity $\left(M_{V, \max } \sim-9.5 \mathrm{mag}\right)$ with the number of clusters brighter than $M_{V} \sim-8.5 \mathrm{mag}\left(N\left(M_{V}<-8.5\right)=13\right)$, as proposed by Gieles et al. (2006a, see also the references therein), we find that the luminosity of the brighter cluster in NGC 3370 can be accounted for by a size-of-sample effect, with a single power-law distribution (see Fig. 16 in Gieles et al. 2006a).

The consistency of the SCLF with a power law indicates no preferred scale for cluster luminosity (i.e. mass). However, "passive" evolutionary processes like tidal interactions, dynamical friction, and the "active" evolution of stars in the cluster are expected to alter the shape of the SCLF. On the contrary, the luminosity function of GCs (GCLF) in both elliptical and spiral galaxies shows a nearly universal turn-over at $M_{V} \sim-7.4 \mathrm{mag}$

\footnotetext{
6 The average $f$ between annuli \#2, \#3, and \#4 at the faint limit is larger than 0.8 in all bands.
}

(Harris 2001; Richtler 2003), implying that present GCs have a preferred mass scale. By inspecting the data in Fig. 13, we do not see any peculiarity near the regions of the GCLF peaks ( $m_{V} \sim 25 \mathrm{mag}$, and $m_{I} \sim 24 \mathrm{mag}$ ), although this might well be a consequence of the poor number of GC candidates in our final catalogue (Sect. 3.5) and the dominance of young objects.

One more piece of information comes from the study of the correlation between the SCLF and Star Formation Rate (SFR) of the galaxy. As shown by different authors (e.g. Larsen \& Richtler 2000; Billett et al. 2002; Larsen 2002), some properties of the SC system are correlated to the SFR, with the efficiency of cluster formation being larger in galaxies with higher SFR. In particular, Larsen (2002) analysed the maximum luminosity expected from a random sampling of the LF (assuming a power law exponent $\alpha=-2.4$ ) versus the SFR per unit area. The comparison of predictions to observations showed a satisfactory agreement. Using the LF shown in Fig. 13 we find that the maximum observed SC candidate magnitude is $M_{V}^{\max } \sim-9.5 \mathrm{mag}$. Assuming the SFR $=0.08 M_{\odot} \mathrm{yr}^{-1}$ by Grosb $\varnothing 1$ \& Dottori (2008, a "crude calculation" based on the $K$-band properties of bright knots in the galaxy), and an area $A \sim 240 \mathrm{kpc}^{2}$, we find that NGC 3370 data fall in the same observational regions as the spirals in Fig. 16 of Larsen (2002), without any noticeable scatter with respect to other observational data. Moreover, the behaviour with respect to the predictions presented in Larsen (2002), based on sampling statistics, is offset by nearly the same amount ( $\sim 0.9$ mag, or $\sim 0.7$ mag, depending on the model) as other spirals.

\subsection{About effective radii and spatial distribution}

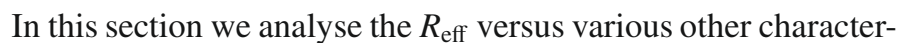
istics of the SC candidates, or the host environment. Let us first inspect the $R_{\text {eff }}$ distribution of the system.

Upper panel of Fig. 14 shows the distribution of $R_{\text {eff }}$ using both the radii obtained from the weighted average of $V$ and $I$ values (solid histogram), and those obtained using all $B V I$ data (dotted line). The differences between the two distributions is negligible, mainly because the $B$-band $R_{\text {eff }}$ have larger uncertainties, thus lower weights. As anticipated, we adopted the $V$ and $I$ averaged $R_{\text {eff }}$ values.

The lower panel of Fig. 14 shows the distribution of $R_{\text {eff }}$ in logarithmic scale. A power law fit to the data (solid line) for $R_{\mathrm{eff}}$ larger than $\sim 3 \mathrm{pc}$, provides an exponent $\eta=-2.35 \pm 0.22$. Such value is consistent with similar results for other galaxies. For example, Bastian et al. (2005) found $\eta=-2.2 \pm 0.2$ for the star clusters in M 51, although they fit with the same power law the whole set of $R_{\text {eff }}\left(2 \mathrm{pc} \leq R_{\text {eff }} \leq 15 \mathrm{pc}\right.$ ). In Fig. 14 it is shown also that a log-normal distribution with a peak at $\sim 3$ pc (dotted line) provides a good fit to data.

The median $R_{\text {eff }}$ of the system is 4.2 , with 3.0 pc standard deviation. If the three possible sub-populations of SC candidates (Sect. 3.1) are taken separately, we obtain as median and standard deviation $R_{\mathrm{eff}}=2.9 \pm 1.5,3.8 \pm 1.8,5.5 \pm 3.2 \mathrm{pc}$ for the blue/intermediate/red peak, respectively (using only the SC candidates within $1 \sigma$ the colour intervals reported in Fig. 10, the number of objects used are 7, 72, and 18 from blue to red peak $^{7}$ ). Although the three median $R_{\text {eff }}$ agree within $1 \sigma$, we notice smaller $R_{\mathrm{eff}}$ for bluer SC candidates. Figure 15 gives more details on this regard. In the figure we show the average $R_{\text {eff }}$ per colour bin for both $B-I$ and $B-V$. In spite of the large scatter,

\footnotetext{
7 We obtain $R_{\text {eff }}=3.3 \pm 1.1,3.8 \pm 2.1, \quad 5.3 \pm 3.5 \mathrm{pc}$ for the blue/intermediate/red peak using SC candidates within $2 \sigma$ the colour intervals of Fig. 10.
} 

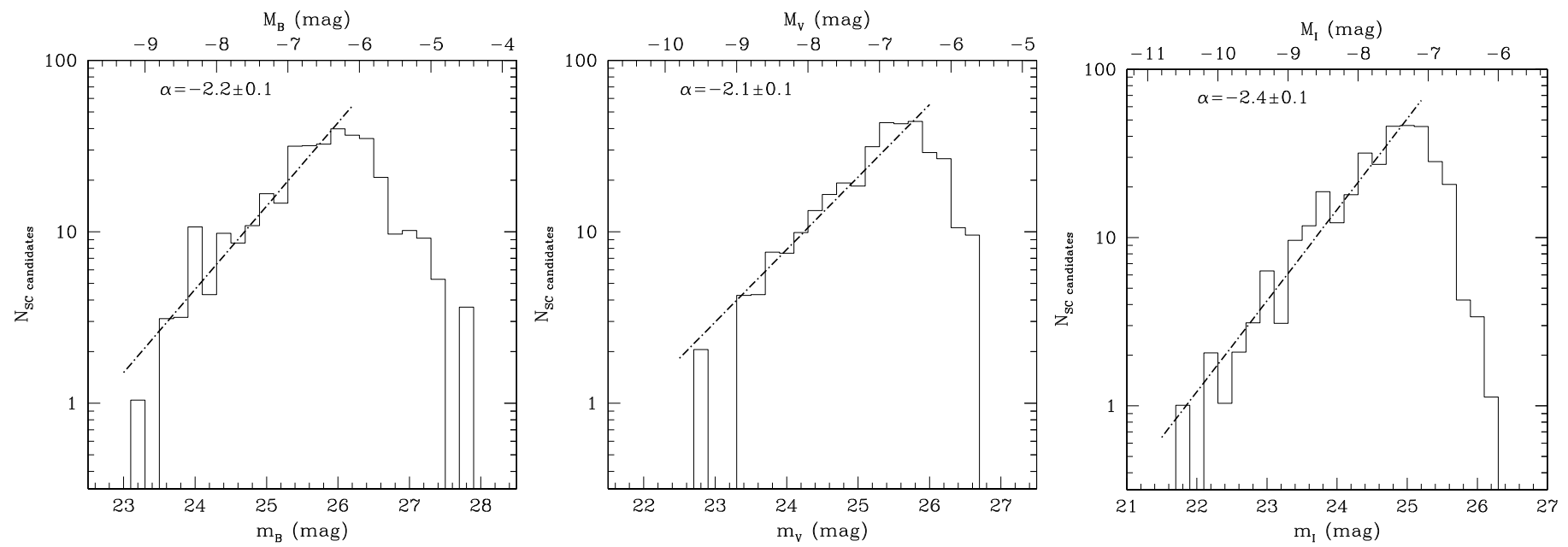

Fig. 13. Left panel: $B$-band Luminosity Function of SC candidates. The dot-dashed line shows the power law fit to the data. The exponent of the fit is also reported in the panel. Middle panel: as upper panel, but for $V$-band data. Right panel: as upper panel, but for I-band data.
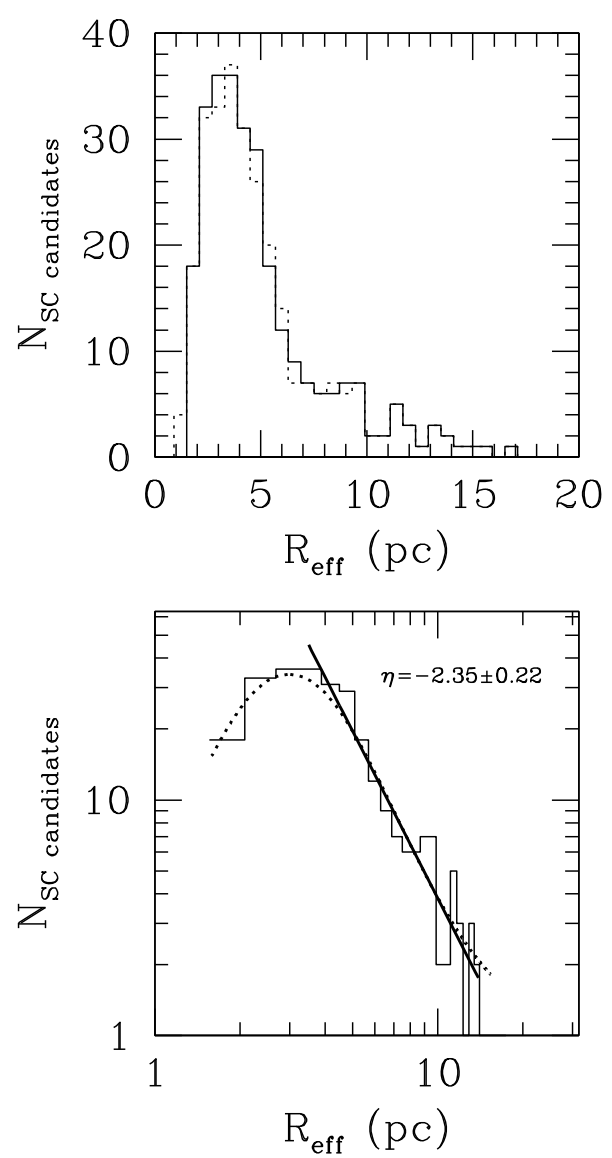

Fig. 14. Upper panel: $R_{\text {eff }}$ distribution for SC candidates. Solid line histogram shows the mean $R_{\text {eff }}$ obtained from $I$ and $V$ band data, the dashed-line shows the histogram obtained coupling $B$-, $V$ - and $I$-band data. Lower panel: as upper panel but in log scales. The thick lines show a power law (only for $R_{\mathrm{eff}} \gtrsim 3 \mathrm{pc}$, solid line), and a log-normal fit to the data (all $R_{\text {eff }}$, dotted line).

it is possible to observe the presence of a non negligible correlation between the size and the colour of SC candidates, with red SCs showing on average larger $R_{\text {eff }}$ respect to blue candidates. Such effect has already been observed in other SC systems (e.g. Scheepmaker et al. 2007). Again, if colour differences are associated to age differences, then the increase of $R_{\text {eff }}$ with colour

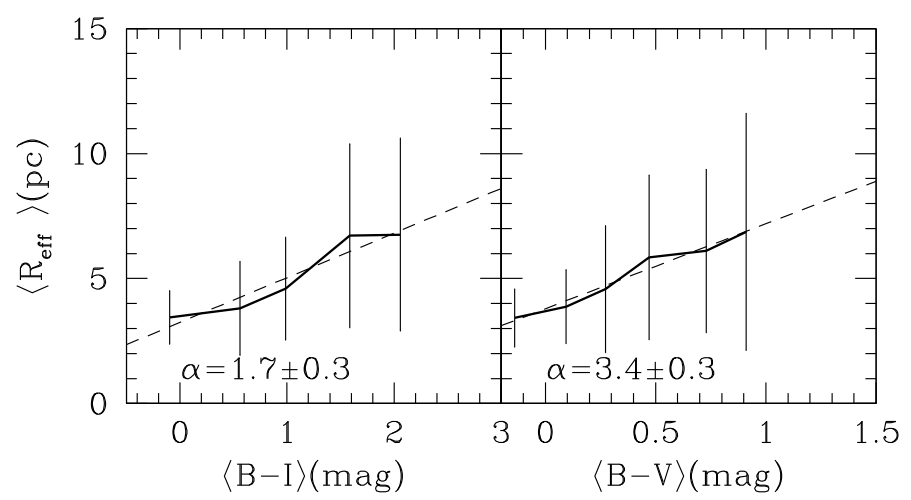

Fig. 15. Median $R_{\text {eff }}$ estimated per colour bins. The slope of the linear fit to data and its rms are reported in the panels.

points toward a dynamical evolution of clusters radii with age, with younger objects being more concentrated than older ones.

The spatial distribution of star clusters provides an additional piece of information on the properties and origins of the $\mathrm{SC}$ system and the host galaxy. One possible clue comes from the relation between the half-light radius and the galactocentric distance $R_{\mathrm{gc}}$, shown in Fig. 16. Also in this case, the spread around the average $R_{\mathrm{eff}}$ for each $R_{\mathrm{gc}}$ bin is large, but a correlation over the entire $R_{\mathrm{gc}}$ range seems to be present. A linear fit to the data provides a slope $\alpha \sim 0.30$. By analysing the SC system of M 51, Scheepmaker et al. (2007) found a shallower slope, $\alpha=0.12 \pm 0.02$. However, similarly to M 51, we find no correlation, or a very weak one, between the colour of the SC candidate and $R_{\mathrm{gc}}$ (Fig. 17). Finally, the spatial density of SC candidates versus $R_{\mathrm{gc}}$, shown in Fig. 18, reveals that the surface brightness profile of the galaxy (in the figure with an arbitrary shift) agrees with the radial density profile of SC candidates. In the figure we omit the central $\sim 2 \mathrm{kpc}$ as SC candidates in such bright region are rejected. A linear fit to SC data provides a slope $\alpha_{\mathrm{SC}}=-0.31 \pm 0.02$, very similar to the $\alpha_{\text {galaxy light }}=-0.28 \pm 0.02$ from the surface brightness profile of the galaxy. Such behaviour, typically observed in late type galaxies (Schweizer et al. 1996; Miller et al. 1997; Larsen 1999), might be an indication that field stars and star clusters have experienced similar dynamical relaxation processes and, that star clusters at any location (and age?) are in dynamical equilibrium with the galaxy potential. 


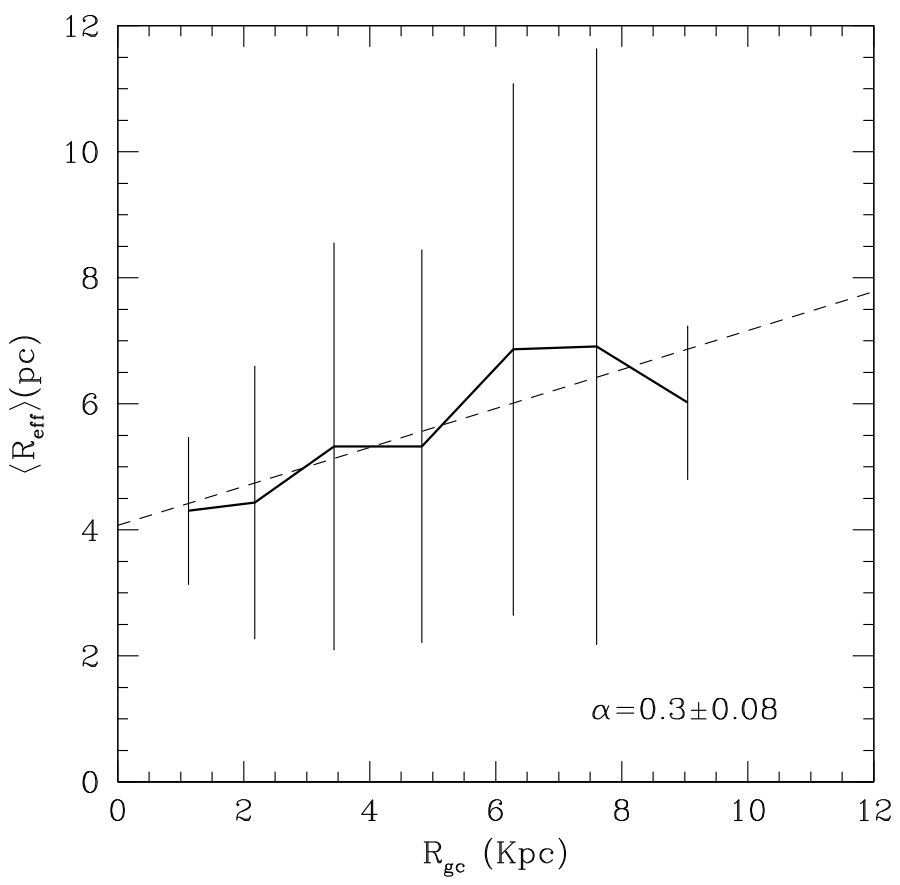

Fig. 16. Average effective radius versus distance from the galaxy centre.

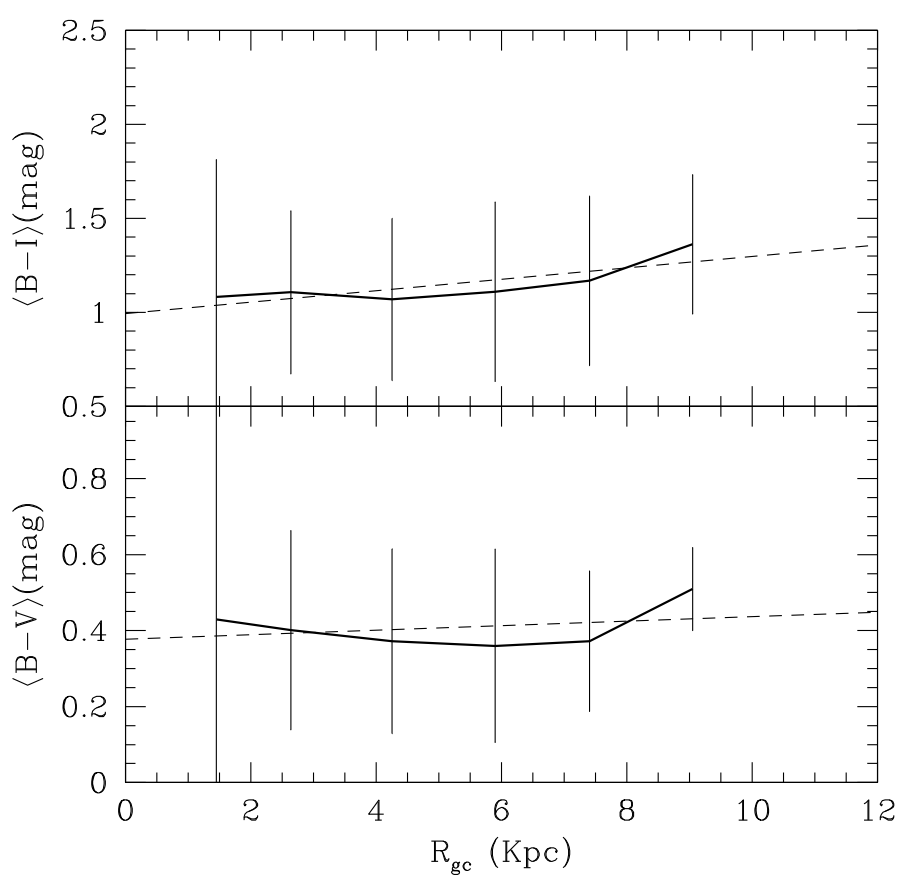

Fig. 17. Average colour of SC candidates versus galactocentric distance.

\subsection{Mass function from comparison with models}

Mass estimates of SCs are sensitive to the age of the cluster, because the mass luminosity ratio changes with time, due to stellar evolution, and because of the dynamical evolution of cluster itself (tidal disruption, evaporation, etc.). In the approximation of no dissolution, Gieles et al. (2007), using SMC data, find that the minimum detectable cluster mass scales with age $t$ according to $M_{\min } \propto t^{+0.7}$, or, in other words, the mass detection limit increases by a factor of $\sim 5$ each age dex (see also Boutloukos \& Lamers 2003, for a similar analysis on clusters in the MW and nearby galaxies). As discussed in Sect. 3.1, the present sample of clusters contains both massive old GCs, which have a peaked

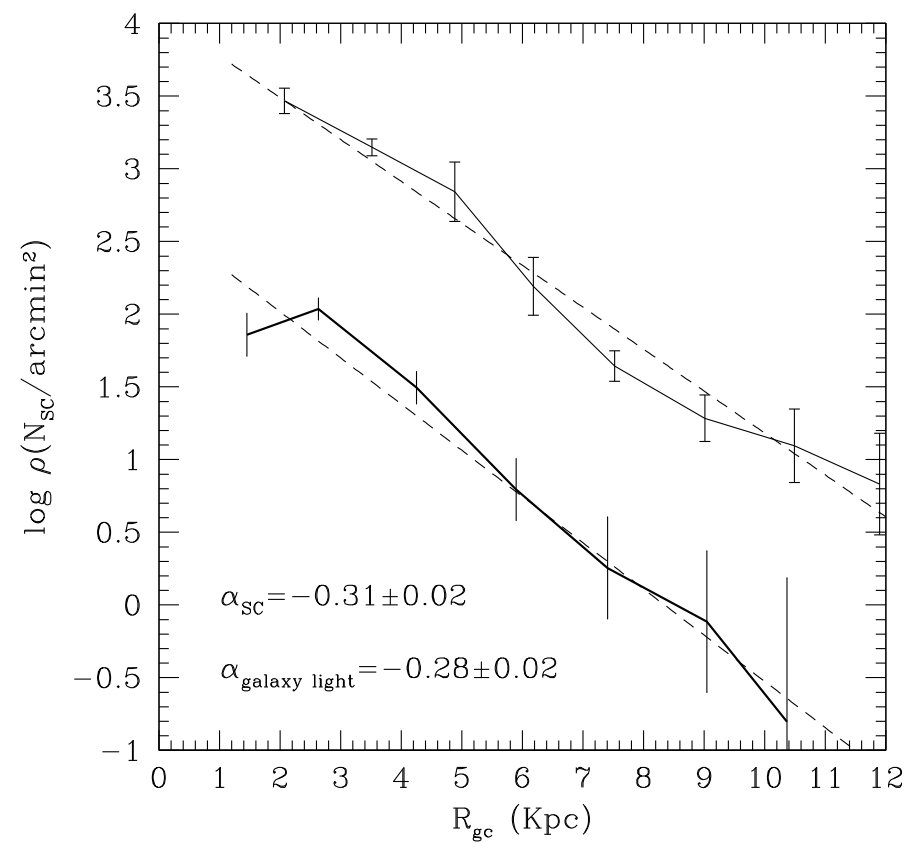

Fig. 18. Radial density of SC candidates corrected for completeness (thick solid line). The completeness correction is only significant in the innermost two bins considered. The upper thin solid line shows the surface brightness profile of the galaxy shifted vertically by an arbitrary amount. The slope of a linear fit to both distributions is also reported in the figure.

mass function, and young clusters, which form from a power-law mass function. Therefore, an age dependent mass-completeness affects our data. To properly constrain the mass of SC candidate, age sensitive data, like $U$-band photometry, are essential, and a study of the SC masses drawn from only BVI data must be considered with extreme caution.

Keeping in mind these warnings, in this section we tentatively derive rough mass estimates of SC candidates. To this purpose we use magnitudes and colours of SC candidates and compare them with stellar population synthesis models. In particular, we take advantage of the specific capabilities of the Teramo-SPoT models (Cantiello et al. 2003; Raimondo et al. $2005)^{8}$, to obtain some hints on the mass distribution of the $\mathrm{SCs}$. Drawing the mass distribution from a comparison of data to SSP models is a risky exercise, and could be misleading if one does not consider properly the limitations due to the assumptions done. The total mass of the SSP strongly depends on the Initial Mass Function. We assumed a Scalo (1998) law; a different IMF, like a Salpeter (1955) or a Kroupa (2001) IMF, would affect the total mass estimates by few tens percent. However, that's rather unimportant for the rough, and relative analysis that we will carry out here. A second approximation comes from the fact that the age and mass associated to each SC candidate depends on the metallicity of the stellar cluster. For this reason, we have assumed three metallicities, representative of metal rich, intermediate, and and metal poor stellar populations, i.e. $[\mathrm{Fe} / \mathrm{H}] \sim-1.8,-0.7$ and $0.0 \mathrm{dex}$, respectively. Then, we have analysed the three cases separately.

To derive crude mass estimates of the SC candidates we used two different approaches, both based on the data to model comparisons: first we compare SC candidates colour and magnitudes with SSP models for the three selected $[\mathrm{Fe} / \mathrm{H}]$ separately, then a

\footnotetext{
8 Visit the website www.oa-teramo.inaf.it/spot for details on models, code, and for downloads.
} 

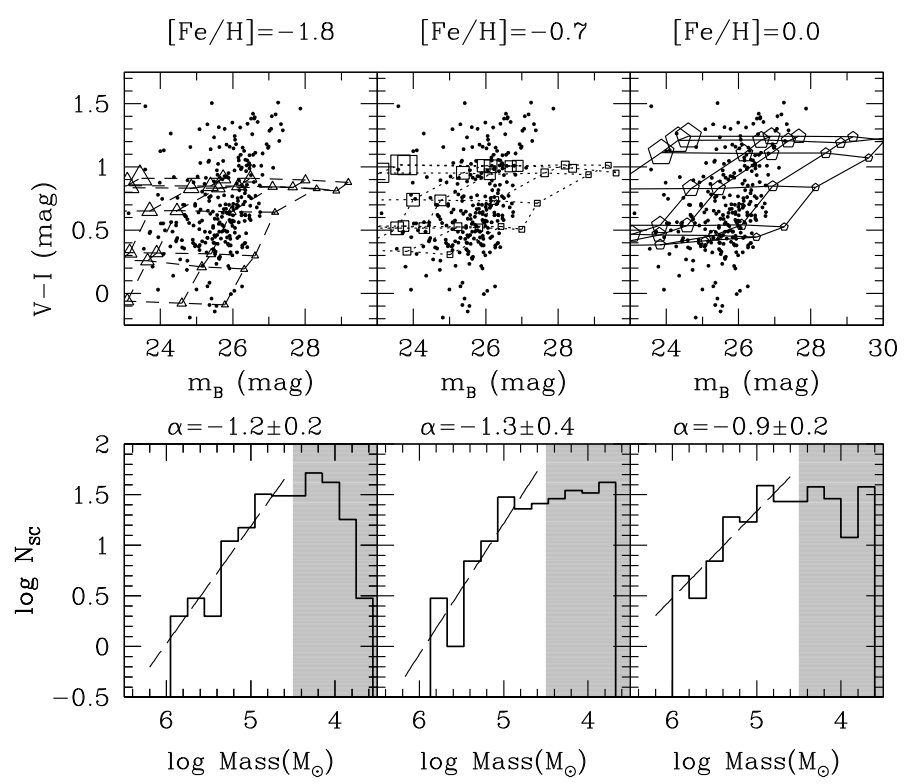

Fig. 19. Upper panels: magnitude-colour diagrams of SC candidates and SPoT SSP models for the three reference metallicities $([\mathrm{Fe} / \mathrm{H}]$ upper quotes in each panel). Models with ages $0.03,0.2,0.5,1,5,10$ and $14 \mathrm{Gyr}$ are connected with a line, older ages have redder colours. SSPs models with different masses, $M_{\text {tot }} \sim 5 \times 10^{3}, 1.5 \times 10^{4}, 6.0 \times 10^{4}, 1.2 \times$ $10^{5}, 1.0 \times 10^{6} M_{\odot}$, are shown with increasing size symbols, depending on the mass. Models are shifted to the distance modulus of NGC 3370. Lower panels: rough estimates of the Present Mass Functions derived from the data to model comparison shown in the upper panels. A linear fit to histograms in the range $4.5 \leq \log \left(M / M_{\odot}\right) \leq 6.5$ is shown with a long-dashed line. The slope of the linear fit is upper quoted in each panel. The shaded region marks, approximatively, the mass interval corresponding to a photometric completeness below $\sim 90 \%$.

second estimate of masses is obtained in the colour-colour plane from median mass-luminosity ratios.

For the specific purpose of this work we have computed SSP models assuming different total masses, i.e. $5.0 \times 10^{3}, 1.5 \times$ $10^{4}, 6.0 \times 10^{3}, 1.2 \times 10^{5}, 1.0 \times 10^{6} M_{\odot}$, and ages $0.03,0.2$, $0.5,1.0,5.0,10.0,14.0 \mathrm{Gyr}$, for the three adopted metal contents. The upper panels in Fig. 19 show a comparison of data to models for the selected $[\mathrm{Fe} / \mathrm{H}]$ values, all computed ages and masses are shown.

Assuming a defined $[\mathrm{Fe} / \mathrm{H}]$, we used the SSP grids shown in Fig. 19 to constrain the mass of the SC candidate, rejecting all SC candidates that lie outside all SSP grids by more than $\sim 0.2$ mag. The lower panels of Fig. 19 show the mass distributions derived from the data-model comparisons described.

Although we cannot give solid constraints on the present mass function (PMF) of SCs, it is instructive to study some features of the magnitude-colour diagrams and PMF derived. First, a few very blue $\left[V-I \lesssim 0.2 \mathrm{mag}, m_{B, 0} \gtrsim 25 \mathrm{mag}\right] \mathrm{SC}$ candidates can only be associated to a metal poor and young component, since intermediate $[\mathrm{Fe} / \mathrm{H}]$ and metal rich models do not provide the necessary colour spread to match these sources. Moreover, the position in the magnitude-colour diagram of these sources implies low masses, $M \lesssim 10^{4} M_{\odot}$. This is also recognised by the presence of a peak at $\sim 10^{4} M_{\odot}$ in the PMF at $[\mathrm{Fe} / \mathrm{H}]=$ -1.8 , while the PMF derived from other $[\mathrm{Fe} / \mathrm{H}]$ are nearly flat below $\sim 10^{5} M_{\odot}$. The expected age-dependent mass completeness (Gieles et al. 2007) is probably observed in the panels of Fig. 19, too. In fact, only young SC candidates overlap with SSP models with masses below $\sim 1.5 \times 10^{4} M_{\odot}$, while no SC candidate matches with the grid of low-mass $t \gtrsim 1$ Gyr SSP models,
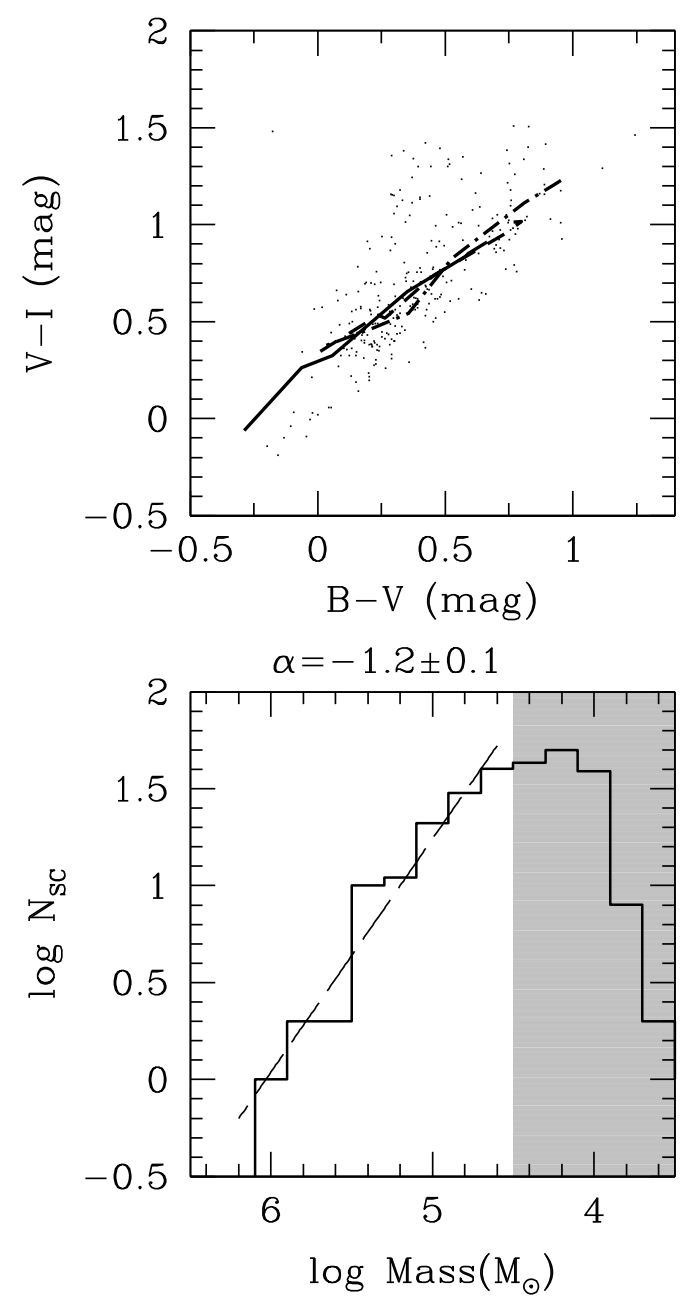

Fig. 20. Upper panel: SC candidates (dots) compared to SSP colour predictions from SPoT for $[\mathrm{Fe} / \mathrm{H}]=-1.8,-0.7,0.0$ (shown with solid, dashed and dot-dashed lines, respectively). The models age ranges from 0.03 to 14 Gyr. Lower panel: the PMF derived from the estimate of median $M / L_{V}$ ratios of $\mathrm{SC}$ candidates.

no matter what $[\mathrm{Fe} / \mathrm{H}]$ is adopted. A similar case are the red objects at $V-I \sim 1.2$, which can only be associated to a population of old, metal rich SCs. Although the sharp drop of the PMFs at masses $\lessgtr 10^{4} M_{\odot}$ might be an artifact of the sample incompleteness, it is worth to note that in all cases a turn over of the PMF around $\sim 10^{5} M_{\odot}$ is observed. Finally, a linear fit to the mass distribution, within the range of $\sim 10^{4.5} M_{\odot} \div \sim 10^{6.5} M_{\odot}$, provides a slope that agrees within $1 \sigma$ uncertainty ${ }^{9}$.

As an additional test we have obtained masses by using the average mass-luminosity $\left(M / L_{V}\right)$ ratios derived from the $B-V$ versus $V-I$ colour-colour diagram, then converting $\mathrm{V}$-band magnitudes to masses. More in details, we have constrained the mean $M / L_{V}$ of the SC candidates by the comparison of SC colours with SSP models in a broad range of ages $(0.03 \leq t(\mathrm{Gyr}) \leq 14.0)$ and $[\mathrm{Fe} / \mathrm{H}]$ from -2.3 to +0.3 dex. We set a default 0.25 "colour distance" to the SSP model for accepting its $M / L_{V}$ ratio $^{10}$. The upper panel of Fig. 20 shows the

9 The lower limit to $\sim 10^{4.5} M_{\odot}$ has been chosen to avoid the masses, i.e. magnitudes, most affected by incompleteness. The $B$-band completeness at masses approximatively lower than $\sim 10^{4.5} M_{\odot}$ drops quickly below $90 \%$.

10 We made several tests using different ranges (from 0.1 to 0.5 mag, also releasing the matching between colours), without any substantial change in the PMF slope. 
colour-colour diagram of SC candidates, and the three reference SSP models from SPoT. The median $M / L_{V}$ obtained from the data-to-models comparison was then used, together with visual magnitude of the SC candidate, to estimate the mass. The main difference with this new approach is that no a priori assumption is done on the $[\mathrm{Fe} / \mathrm{H}]$ content. Moreover, in the previous approach, at fixed $[\mathrm{Fe} / \mathrm{H}]$ the mass and age of the SC were virtually present in the comparisons shown in Fig. 19 (upper panels), while in this case mass comes from the statistical analysis of the $M / L_{V}$ of SSP models within large age and $[\mathrm{Fe} / \mathrm{H}]$ ranges. The PMF derived using $M / L_{V}$ ratios is shown in Fig. 20. Our main interest, here, is to emphasise that the two approaches used to derive the PMF provide similar results concerning the power law profile.

The PMF in spiral and irregular galaxies is generally well fit by a power-law, although the fit is limited to the young and most massive component of clusters. The typical value for power law exponent is $\alpha \sim-2.0$ (e.g. Zhang \& Fall 1999; Bik et al. 2003). Similarly, the high mass tail of Galactic OCs has a slope $\alpha=$ $-2.04 \pm 0.11$, with no selection on clusters age, thus including objects from few Myr up to several Gyr (Battinelli et al. 1994).

Such numbers agree with the slopes obtained from the power-law fit to data (shown in Figs. 19, 20), ranging between $-1.3 \pm 0.4$ and $-0.9 \pm 0.2$, and corresponding to a power law index $\alpha=-2.3 \div-1.9$ in the form $\mathrm{d} N_{\mathrm{SC}} / \mathrm{d} M \propto M^{\alpha}$.

Recently, Larsen (2009) proposed that the power-law fit to the MF of SCs might be due to the limited mass range of observational data, and suggested that a functional form of the type of a Schechter law - characterised by a power law and an exponential decline after some characteristic mass $M_{\mathrm{c}} \sim 2.1 \times 10^{5} M_{\odot}$ - approximates quite well some of the best studied SC systems, like in M 51 and LMC. However, Larsen also warns that it is unlikely that such functional form is universal, quoting the cases of some merger or starburst galaxies as examples where significantly higher $M_{\mathrm{c}}$ values are required. As a non-interacting spiral, NGC 3370 should fall in the sample of galaxies with a PMF well described by a Schechter form. Assuming a Schecter function as in Larsen (2009), a cut-off mass of the order of $M_{\mathrm{c}} \sim 10^{6} M_{\odot}$ would be required for the approximate PMF derived here. Such high $M_{\mathrm{c}}$ value has been fit to the young SCs in the Antennae (Jordan et al. 2007), but it is highly unlikely in spirals, being probably a result of the sample incompleteness at low masses, and the rough mass estimates used here.

\subsection{Globular Cluster candidates}

The colour distributions of NGC 3370 clusters, and the comparisons with GGCs, as well as with the cluster system in other galaxies, shown in previous sections, has evidenced the presence of a red system of objects with properties remarkably similar to old globular clusters. In this section we provide a more detailed study on such GC candidates. To select a sample of candidate GCs we consider only sources with $V-I$ matching with the GC system in the Galaxy, in addition sources in the brightest/bluest part of the disk are rejected to avoid contamination $\left(R_{\mathrm{gc} \min } \geq 50^{\prime \prime}, \mu_{V} \geq 24.0 \mathrm{mag} / \operatorname{arcsec}^{2}\right)$. With such criteria we end up with a list of 35 GC candidates. Figure 21 shows the colour histograms (normalised to the maximum bin amplitude) for GCs in NGC 3370 and, for comparison, for GGCs brighter than $M_{V} \gtrsim-5$ (GGC data from Harris 1996). The median colours are $B-V=0.62 \pm 0.15 \mathrm{mag}(0.68 \pm 0.11 \mathrm{mag})$, and $B-I=1.60 \pm 0.27 \mathrm{mag}(1.58 \pm 0.19 \mathrm{mag})$ for NGC 3370 (MW) star clusters. The median $R_{\text {eff }}$ is $\sim 5.5$ with 3.9 pc standard deviation. Having in mind the warning of the small sample of

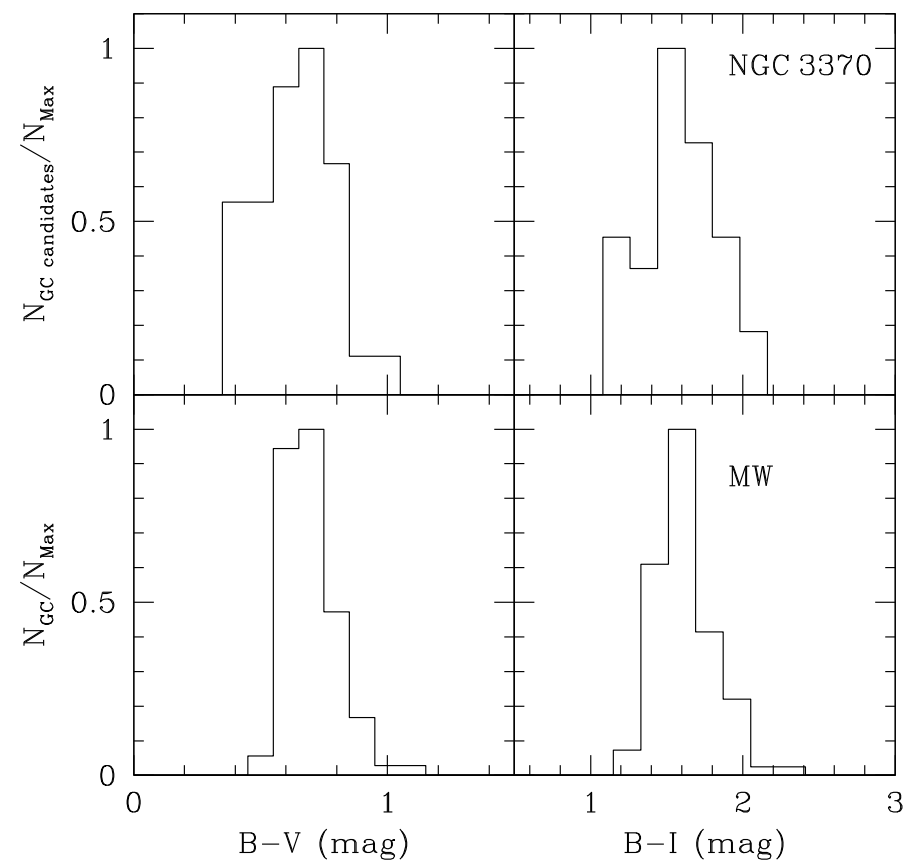

Fig. 21. Colour histograms of GC candidates in NGC 3370 (upper panels) and of bright GGCs (lower panels). The histograms are normalised to the bin with maximum amplitude.

GC selected, it is possible to associate the similarity between the $B-V$ and $B-I$ colour distributions in both galaxies to similar age/metallicity properties of the two GC systems. However, some differences seems to be recognised between the two galaxies. First, the colour distributions in NGC 3370 do not appear so sharp as in the case of GGC, though this might be a consequence of larger photometric uncertainties. Second, both NGC 3370 distributions show a tail of GC at bluer colours with respect to the MW - more evident in $B-I$. If the apparent secondary peak is interpreted as a metallicity difference then, using the $B-I$ colour-metallicity relation by Harris et al. (2006) derived from GGCs, we find that the main peak corresponds to a metallicity $[\mathrm{Fe} / \mathrm{H}] \sim-1.5 \mathrm{dex}$ (as for the Galaxy), while the $[\mathrm{Fe} / \mathrm{H}]$ for objects in the blue tail is $\sim-2.3$ dex. However, we must emphasise that several doubts have been raised in the last few years against the simple interpretation of colour bimodalities in GC systems as metallicity bimodalities. Non-linear metallicity-colour transformations, in fact, have been proved to be effective in generating multimodal colour distributions from unimodal $[\mathrm{Fe} / \mathrm{H}]$ distributions (see Cantiello \& Blakeslee 2007, and references therein).

One other useful quantity related to the GC system is the specific frequency, $S_{N}$, i.e. the number of GC normalised to the host galaxy luminosity. This number provides a useful quantity related to the formation mechanisms of the galaxy (see Brodie \& Strader 2006, and references therein). To properly estimate $S_{N}$, however, the total number of GC needs to be used. As a consequence of the sample incompleteness we cannot reliably constrain the specific frequency, but only provide an estimate to the lower limit of $S_{N}$. In particular, we make the following assumptions: $i$ ) we use the results by, e.g., Rhode \& Zepf (2003) showing that the surface density of GC in spirals follows a power law profile; $i$ ) a total apparent corrected magnitude $m_{V, 0} \sim 11.7 \mathrm{mag}$ for NGC 3370 from Hyperleda ${ }^{11}$ is adopted, and iii) a the slope for the density profile of $\log \rho_{\mathrm{GC}} \sim-1.5 \log R_{\mathrm{gc}}$ is fixed from the compilation of Kissler-Patig (1997), for a total galaxy magnitude

${ }^{11}$ http://leda.univ-lyon $1 . f r$ 
$M_{V} \sim 21.5 \mathrm{mag}$. By constraining the intercept of the density profile from the number of GCs within $50^{\prime \prime} \div 80^{\prime \prime}-$ corrected for photometric completeness - we find a lower limit of $S_{N} \sim 0.8$, to be compared with $S_{N} \geq 0.5$ for the GC systems on other spirals (Kissler-Patig 1997; Rhode \& Zepf 2003; Chandar et al. 2004).

\section{Summary}

Deep optical $V I$ and shallower $B$ band observations of the face on spiral galaxy NGC 3370, drawn from the ACS/HST archive, have been used to study the SC system in the galaxy. The characteristics of the imaging data available allowed to analyse the photometry of SC candidates, and their spatial properties. The final list of SC candidates consists of 277 objects, selected according to maximum colour, magnitude, size and position within the galaxy.

The analysis to the sample of SC candidates leads to the following conclusions:

(1) The colour distributions show that the SC population is composed by different subpopulations. In particular, the differences are related to ages, with the main sub-populations represented by $i$ ) a red system of likely GC candidates; ii) a dominant peak of sources with properties similar to intermediate age clusters (similar to OCs with ages from a few 100 Myrs, to few Gyr); and iii) a blue tail of SCs, possibly with ages below 100 Myr. Such complex age-structure of the SC system is somehow expected in a Sc-type spiral, actively star-forming (as also testifies the amount of Cepheids observed in this galaxy).

(2) The comparison of SC candidates colours - selected with no a priori assumption on the range of colours allowed - with SSP models predictions within a large range of ages (30 Myr to $14 \mathrm{Gyr})$ and $[\mathrm{Fe} / \mathrm{H}](-2.3$ to $+0.3 \mathrm{dex})$ shows a good matching between models and data. A significant exception to this is represented by a sample of $\sim 20$ SC candidates, which systematically lie outside the region of SSP models, showing an excess of flux at longer wavelength (I-band magnitude). Such SC candidates do not show remarkable peculiarities in terms of spatial distributions or morphology. One possible origin of their behaviour is that they are typical intermediate age SCs (300 Myr to 2 Gyr). At these ages, the stochastic presence of TP-AGB stars has a dramatic impact on the integrated light. In particular, TP-AGBs dominate the long-wavelength emission of the stellar system (see, e.g., Maraston 2005; Marigo et al. 2008, and references therein), thus the stochastic presence of TP-AGBs might cause the observed position of these SCs in the colour-colour diagram (Raimondo 2009).

(3) The median $R_{\text {eff }}$ of the system is 4.2 with 3.0 pc standard deviation. The $R_{\text {eff }}$ distribution shows a power law drop above $R_{\text {eff }} \sim 3.0 \mathrm{pc}$. The exponent of the power law, $\eta=2.35 \pm 0.22$, appears normal with respect to other similar galaxies. Moreover, a log-normal fit to the distribution of $R_{\text {eff }}$ seems to show a turn-over around $\sim 3 \mathrm{pc}$, although the completeness of the data does not allow to give more robust conclusions.

(4) The $R_{\text {eff }}$ shows non-negligible correlations both with the colour of the SC candidate, and with the galactocentric distance $R_{g c}$. If colours differences are associated to age differences, as suggested in (1), these results point toward an age evolution of $R_{\text {eff }}$, with older SCs having larger $R_{\text {eff }}$. Furthermore, the spatial density of clusters, which appears similar to the surface brightness profile of the galaxy, might well be a consequence of a common dynamical evolution of stars and star clusters in the gravitational potential of the galaxy.

(5) The LF of SC candidates shows a continuous increase down to magnitudes where the SC candidate sample is fairly unaffected by incompleteness. Such LF is well described by a power law with exponent slightly below $\sim-2.0$ in all bands. This functional form agrees to that of other SC systems in spiral or irregular galaxies. A comparison of the maximum SC magnitude with the SFR per unit area shows that the correlation between these quantities is like in other spirals. Moreover, some properties of the LFs presented here (steepening of the LF towards brighter magnitudes, and steeper slopes at longer wavelengths) seem to support the idea that the SCLF is originated by a truncated mass function, though sample incompleteness hampers more detailed conclusions.

(6) By comparing data to SSP models, we tentatively derived a crude estimate of the Present Mass Function of the SC system. Since our data suffer for the lack of an age-sensitive indicator, the PMF derived must be taken carefully as a best guess with available data. With this warning in mind, we find that a power law fit to the interval of more massive SCs (i.e. objects likely less affected by the incompleteness) provides an $\alpha \sim-2.3 \div-1.9$, in agreement to what can be found in literature for similar galaxies. Moreover, the PMF seems to show a turn-over around masses of the order of $\sim 10^{5} M_{\odot}$, though this is possibly due to the sample incompleteness at lower masses.

(7) We analysed the properties of a sample of GC candidates, selected on the base of their $V-I$ colour, and their positions with respect to the centre of the galaxy. The number of GC candidates selected is 35 , these show colour distributions similar to GGCs, though less peaked around a median value (probably due to the larger photometric uncertainties), and with a relatively more populated tail towards bluer colours. The peak of the colour distribution, converted to metallicity using GGCs data, gives $[\mathrm{Fe} / \mathrm{H}] \sim-1.5$, similar to GGCs.

(8) Due to the complex issue of completeness in these data, we cannot provide a reliable estimate to the specific frequency of GCs. However, taking advantage of the known approximative behaviour of the surface density profile of GC in spirals, we estimate a lower limit $S_{N}>0.8$.

The high resolution of ACS/WFC allowed us to carry on a refined study of the structural and photometric properties of the SC system in NGC 3370. These data are essential to provide further constraints to the evolution of stars and stellar systems in this galaxy. On the other hand, the lack of age-sensitive indicators, like shorter-wavelength ( $\sim U$-band) photometry, is a serious drawback for a detailed study of ages and masses of the SC system in this galaxy. The restoration of ACS and the installation of WFC3 on the HST, after SM4, will certainly help to fill the present lack of data.

Acknowledgements. It is a pleasure to thank Gabriella Raimondo for several scientific discussions and for her irreplaceable support in computing theoretical models. We are also grateful to Sören Larsen for his fruitful comments and suggestions on data analysis. Part of this work was supported by PRIN-INAF 2006 (PI G. Clementini). We would thank the referee, Dr. Mark Gieles, for valuable remarks and suggestions.

\section{References}

Bastian, N., Gieles, M., Lamers, H. J. G. L. M., Scheepmaker, R. A., \& de Grijs, R. 2005, A\&A, 431, 905

Battinelli, P., Brandimarti, A., \& Capuzzo-Dolcetta, R. 1994, A\&AS, 104, 379 
Bertin, E. \& Arnouts, S. 1996, A\&AS, 117, 393

Bica, E., Claria, J. J., Dottori, H., Santos, Jr., J. F. C., \& Piatti, A. E. 1996, ApJS, 102,57

Bik, A., Lamers, H. J. G. L. M., Bastian, N., Panagia, N., \& Romaniello, M. 2003, A\&A, 397, 473

Billett, O. H., Hunter, D. A., \& Elmegreen, B. G. 2002, AJ, 123, 1454

Blakeslee, J. P., Anderson, K. R., Meurer, G. R., Benítez, N., \& Magee, D. 2003, in Astronomical Data Analysis Software and Systems XII, ASP Conf. Ser., 295

Boutloukos, S. G., \& Lamers, H. J. G. L. M. 2003, MNRAS, 338, 717

Brodie, J. P., \& Strader, J. 2006, ARA\&A, 44, 193

Cantiello, M., \& Blakeslee, J. P. 2007, ApJ, 669, 982

Cantiello, M., Raimondo, G., Brocato, E., \& Capaccioli, M. 2003, AJ, 125, 2783

Chandar, R., Whitmore, B., \& Lee, M. G. 2004, ApJ, 611, 220

de Grijs, R., Anders, P., Bastian, N., et al. 2003, MNRAS, 343, 1285

de Vaucouleurs, G., de Vaucouleurs, A., Corwin, Jr., H. G., et al. 1991, Third Reference Catalogue of Bright Galaxies (Berlin, Heidelberg, New York: Springer-Verlag), Vol. 1-3, XII, 2069

Elson, R. A. W., \& Fall, S. M. 1985, PASP, 97, 692

Elson, R. A. W., Fall, S. M., \& Freeman, K. C. 1987, ApJ, 323, 54

García-Barreto, J. A., Hernández-Toledo, H., Moreno-Díaz, E., Bernal-Marín, T., \& Villarreal-Castillo, A. L. 2007, AJ, 134, 142

Gieles, M., Larsen, S. S., Bastian, N., \& Stein, I. T. 2006a, A\&A, 450, 129

Gieles, M., Larsen, S. S., Scheepmaker, R. A., et al. 2006b, A\&A, 446, L9

Gieles, M., Lamers, H. J. G. L. M., \& Portegies Zwart, S. F. 2007, ApJ, 668, 268

Grosbøl, P., \& Dottori, H. 2008, A\&A, 490, 87

Harris, W. E. 1996, AJ, 112, 1487

Harris, W. E. 2001, in Star Clusters, Saas-Fee Advanced Course 28, ed. L. Labhardt \& B. Binggeli, 223

Harris, W. E., Whitmore, B. C., Karakla, D., et al. 2006, ApJ, 636, 90

Jordan, A., McLaughlin, D. E., Cote, P., et al. 2007, ArXiv Astrophysics e-prints

Kissler-Patig, M. 1997, A\&A, 319, 83

Kroupa, P. 2001, MNRAS, 322, 231

Lada, C. J., \& Lada, E. A. 2003, ARA\&A, 41, 57
Larsen, S. S. 1999, A\&AS, 139, 393

Larsen, S. S. 2002, AJ, 124, 1393

Larsen, S. S. 2009, A\&A, 494, 539

Larsen, S. S., \& Richtler, T. 2000, A\&A, 354, 836

Lata, S., Pandey, A. K., Sagar, R., \& Mohan, V. 2002, A\&A, 388, 158

Mackey, A. D., \& Gilmore, G. F. 2003, MNRAS, 338, 85

Maraston, C. 2005, MNRAS, 362, 799 (Mar05)

Marigo, P., Girardi, L., Bressan, A., et al. 2008, A\&A, 482, 883

McLaughlin, D. E., \& van der Marel, R. P. 2005, ApJS, 161, 304

Miller, B. W., Whitmore, B. C., Schweizer, F., \& Fall, S. M. 1997, AJ, 114, 2381

Park, W.-K., \& Lee, M. G. 2007, AJ, 134, 2168

Rafelski, M., \& Zaritsky, D. 2005, AJ, 129, 2701

Raimondo, G. 2009, ApJ, 700, 1247

Raimondo, G., Brocato, E., Cantiello, M., \& Capaccioli, M. 2005, AJ, 130, 2625

Rhode, K. L., \& Zepf, S. E. 2003, AJ, 126, 2307

Richtler, T. 2003, in Stellar Candles for the Extragalactic Distance Scale, ed.

D. Alloin, \& W. Gieren, Lect. Notes Phys., 635, 281

Riess, A. G., Press, W. H., \& Kirshner, R. P. 1996, ApJ, 473, 88

Riess, A. G., Li, W., Stetson, P. B., et al. 2005, ApJ, 627, 579

Salpeter, E. E. 1955, ApJ, 121, 161

Scalo, J. 1998, in The Stellar Initial Mass Function, 38th Herstmonceux Conference, ed. G. Gilmore, \& D. Howell, ASP Conf. Ser., 142, 201

Scheepmaker, R. A., Haas, M. R., Gieles, M., et al. 2007, A\&A, 469, 925

Schlegel, D. J., Finkbeiner, D. P., \& Davis, M. 1998, ApJ, 500, 525

Schweizer, F., Miller, B. W., Whitmore, B. C., \& Fall, S. M. 1996, AJ, 112, 1839

Searle, L., Wilkinson, A., \& Bagnuolo, W. G. 1980, ApJ, 239, 803

Sirianni, M., Jee, M. J., Benítez, N., et al. 2005, PASP, 117, 1049

van den Bergh, S., \& Morbey, C. L. 1984, ApJ, 283, 598

van Dyk, S. D., Treffers, R. R., Richmond, M. W., Filippenko, A. V., \& Paik, Y. B. 1994, IAU Circ., 6105, 1

Whitmore, B. C., \& Schweizer, F. 1995, AJ, 109, 960

Whitmore, B. C., Zhang, Q., Leitherer, C., et al. 1999, AJ, 118, 1551

Zhang, Q., \& Fall, S. M. 1999, ApJ, 527, L81 